JOURNAL OF THE

AMERICAN MATHEMATICAL SOCIETY

Volume 10, Number 2, April 1997, Pages 393-426

S 0894-0347(97)00231-2

\title{
LOCAL EXACTNESS \\ IN A CLASS OF DIFFERENTIAL COMPLEXES
}

\author{
SAGUN CHANILLO AND FRANÇOIS TREVES
}

\section{Contents}

1. The differential complex under study.

Statement of the theorem

2. The lift to the sphere. Two lemmas. Proof of the theorem 397

3. Proof of Lemma 2.1

4. Proof of Lemma 2.2. Preparatory estimates 413

5. $\quad$ End of the proof of Lemma $2.2 \quad 418$

Appendix: Estimates in the integration of $p$-forms

on a compact manifold $\quad 424$

Acknowledgements $\quad 425$

References $\quad 425$

\section{The DifFERENTIAL COMPlEX UNDER STUDy. STATEMENT OF THE THEOREM}

Let $\mathcal{M}^{n+1}$ be a manifold of class $\mathcal{C}^{\omega}$, and let a real-analytic vector subbundle $T^{\prime}$ of the complex(ified) cotangent bundle $\mathbb{C} T^{*} \mathcal{M}$ be given, which is spanned, locally, by the differential of a single analytic function. The orthogonal $\mathcal{V}$ of $T^{\prime}$ for the duality between tangent and cotangent vectors is a vector subbundle of $\mathbb{C} T \mathcal{M}$ of rank $n=\operatorname{dim} \mathcal{M}-1$, which satisfies the Frobenius condition $[\mathcal{V}, \mathcal{V}] \subset \mathcal{V}$. Conversely, given any real-analytic vector subbundle $\mathcal{V}$ of $\mathbb{C} T \mathcal{M}$ whose fibre dimension (i.e., rank) is equal to $n$ and which is such that $[\mathcal{V}, \mathcal{V}] \subset \mathcal{V}$, its orthogonal $T^{\prime}$ is locally generated by a single closed one-form of class $\mathcal{C}^{\omega}$.

A differential complex on $\mathcal{M}$ is naturally associated to a structure of this type. We quickly recall the coordinate free definition of this complex. Let $\Lambda^{p}\left(\mathbb{C} T^{*} \mathcal{M}\right)$ denote the $p$ th exterior power of $\mathbb{C} T^{*} \mathcal{M}$, and let $\Lambda^{1, q}$ denote the vector subbundle of $\Lambda^{q+1}\left(\mathbb{C} T^{*} \mathcal{M}\right)$ spanned by exterior products of $\omega_{0} \wedge \omega_{1} \wedge \cdots \wedge \omega_{q}(0 \leq q \leq n)$ with $\omega_{0}$ a smooth section of $T^{\prime}$ and $\omega_{1}, \ldots, \omega_{q}$ smooth one-forms in $\mathcal{M}$. We have $d \omega_{0} \in \Lambda^{1,1}$, hence $d\left(\omega_{0} \wedge \omega_{1} \wedge \cdots \wedge \omega_{q}\right) \in \Lambda^{1, q+1}$. If $\varpi$ is a smooth section of $\Lambda^{1, q}$, then $d \varpi$

Received by the editors May 17, 1996 and, in revised form, November 11, 1996

1991 Mathematics Subject Classification. Primary 35A07, 35F05.

Key words and phrases. Differential complex, local solvability, singular homology, subanalytic sets.

The first author was partially supported by NSF Grant DMS-9401782, and the second author by NSF Grant DMS-9201980.

(C)1997 American Mathematical Society 
is one of $\Lambda^{1, q+1}$. Set $\Lambda^{0, q}=\Lambda^{q}\left(\mathbb{C} T^{*} \mathcal{M}\right) / \Lambda^{1, q-1}$; if a smooth form $\varpi$ represents a section of $\Lambda^{0, q}$, then $d \varpi$ represents one of $\Lambda^{0, q+1}$. We denote by $\mathcal{C}^{\infty}\left(U ; \Lambda^{p, q}\right)$ [resp., $\left.\mathcal{D}^{\prime}\left(U ; \Lambda^{p, q}\right)\right]$ the space of $\mathcal{C}^{\infty}$ (resp., distribution) sections of $\Lambda^{p, q}$ over an open subset $U$ of $\mathcal{M}(p=0,1)$. Of course $\mathcal{C}^{\infty}\left(U ; \Lambda^{0,0}\right)=\mathcal{C}^{\infty}(U), \mathcal{D}^{\prime}\left(U ; \Lambda^{0,0}\right)=\mathcal{D}^{\prime}(U)$ and $\mathcal{D}^{\prime}\left(U ; \Lambda^{p, q}\right)=\{0\}$ if $q>n$. The exterior derivative $d$ induces differential operators

$$
d^{\prime}: \mathcal{D}^{\prime}\left(U ; \Lambda^{p, q-1}\right) \rightarrow \mathcal{D}^{\prime}\left(U ; \Lambda^{p, q}\right), \quad d^{\prime}: \mathcal{C}^{\infty}\left(U ; \Lambda^{p, q-1}\right) \rightarrow \mathcal{C}^{\infty}\left(U ; \Lambda^{p, q}\right) .
$$

Clearly $d^{2}=0 \Rightarrow d^{2}=0$. For $p=0,1$ fixed and $q=1,2, \ldots$, we get two differential complexes on $U \subset \mathcal{M}$.

In well-chosen local coordinates one can get concrete and convenient representations of these differential complexes. In a suitably small open neighborhood $\Omega$ of an arbitrary point $O$ of $\mathcal{M}$ we can select a $\mathcal{C}^{\omega}$ function $Z$ whose differential $d Z$ spans $\left.T^{\prime}\right|_{\Omega}$, and real-analytic coordinates $x, t_{1}, \ldots, t_{n}$ vanishing at $O$ and such that $\left.Z\right|_{t=0}=x$. Possibly after contracting $\Omega$ about $O$, we change our choice of the coordinate $x$ to $x=\operatorname{Re} Z$. These coordinates identify $\Omega$ to an open subset of $\mathbb{R}^{n+1}$ in which

$$
Z(x, t)=x+\iota \Phi(x, t) \quad(\iota=\sqrt{-1}),
$$

with $\Phi \in \mathcal{C}^{\omega}(\Omega)$ real-valued and $\left.\Phi\right|_{t=0}=0$. The vector fields $L_{1}, \ldots, L_{n}$ defined by the relations

$$
L_{j} Z=0, \quad L_{j} t_{k}=\delta_{j k} \quad(j, k=1, \ldots, n)
$$

form a basis of $\left.\mathcal{V}\right|_{\Omega}$. This will be our framework throughout the remainder of the article. The point $O$ becomes the origin of $\mathbb{R}^{n+1}$.

If $z_{0} \in \mathbb{C}$ and if $S$ is a subset of $\Omega$, then we shall refer to the pre-image $\mathcal{F}\left(z_{0}, S\right)=$ $\left\{(x, t) \in S ; Z(x, t)=z_{0}\right\}$ as a fibre of $Z$ in $S$. Of course, $\mathcal{F}\left(z_{0}, S\right)=\varnothing$ if $z_{0} \notin Z(S)$. The germs of sets at $O$ represented by the fibres of $Z$ in neighborhoods of $O$ are invariants of the locally integrable structure defined by $T^{\prime}$ and $\mathcal{V}$ (Baouendi-Treves [1]; also Treves [20, Corollary II.3.1]).

It is also convenient to introduce the vector field $L_{0}$ defined by

$$
L_{0} Z=1, \quad L_{0} t_{k}=0 \quad(k=1, \ldots, n) .
$$

Of course the vector fields $L_{0}, L_{1}, \ldots, L_{n}$ form a basis over $\Omega$ of the complex tangent bundle. The relations (1.2) and (1.3) entail $\left[L_{j}, L_{k}\right]=0$ for all $j, k=0,1, \ldots, n$. The coefficients of the vector fields $L_{j}$ are complex-valued and real-analytic. Actually,

$$
L_{0}=\left(1+\iota \Phi_{x}\right)^{-1} \partial / \partial x, \quad L_{j}=\partial / \partial t_{j}-\iota\left(\partial \Phi / \partial t_{j}\right) L_{0}, \quad j=1, \ldots, n .
$$

Notice that if $f \in \mathcal{C}^{\infty}(\Omega)$, then

$$
d f=L_{0} f d Z+\sum_{j=1}^{n} L_{j} f d t_{j}
$$

If $U$ is an open subset of $\Omega$ and $q$ a positive integer, the space $\mathcal{C}^{\infty}\left(U ; \Lambda^{0, q}\right)$ (see the beginning of this section) can and will be identified to the space of differential forms

$$
f(x, t, d t)=\sum_{|I|=q} f_{I}(x, t) d t_{I},
$$

where $f_{I} \in \mathcal{C}^{\infty}(U), I=\left\{i_{1}, \ldots, i_{q}\right\} \in \mathbb{Z}_{+}^{q}$ with $1 \leq i_{1}<\cdots<i_{q} \leq n$, and

$$
d t_{I}=d t_{i_{1}} \wedge \cdots \wedge d t_{i_{q}}
$$


As for the elements of $\mathcal{C}^{\infty}\left(U ; \Lambda^{1, q}\right)$ they are the differential forms $f \wedge d Z$ with $f$ as in (1.6). Of course, $f \rightarrow f \wedge d Z$ is an isomorphism of $\mathcal{C}^{\infty}\left(U ; \Lambda^{0, q}\right)$ onto $\mathcal{C}^{\infty}\left(U ; \Lambda^{1, q}\right)$.

The vector fields $L_{j}$ define an operator $L$ acting on forms (1.6):

$$
L f(x, t, d t)=\sum_{|I|=q} \sum_{j=1}^{n} L_{j} f_{I}(x, t) d t_{j} \wedge d t_{I} .
$$

According to (1.4) we may write

$$
L f=d_{t} f-\iota d_{t} \Phi \wedge L_{0} f,
$$

with the vector field $L_{0}$ acting coefficientwise on $f$. We obtain a differential complex

$$
L: \mathcal{C}^{\infty}\left(U ; \Lambda^{0, q-1}\right) \rightarrow \mathcal{C}^{\infty}\left(U ; \Lambda^{0, q}\right), \quad q=1,2, \ldots,
$$

since $L^{2}=0$. Formula (1.5) shows that

$$
d(f \wedge d Z)=L f \wedge d Z, \quad \forall f \in \mathcal{C}^{\infty}\left(U ; \Lambda^{0, q}\right) .
$$

Thus the differential complex (1.9) is isomorphic to the complex

$$
d: \mathcal{C}^{\infty}\left(U ; \Lambda^{1, q-1}\right) \rightarrow \mathcal{C}^{\infty}\left(U ; \Lambda^{1, q}\right), \quad q=1,2, \ldots
$$

We have analogous complexes of currents, i.e., differential forms with distributions as coefficients; it suffices to substitute $\mathcal{D}^{\prime}$ for $\mathcal{C}^{\infty}$ in what precedes.

One commonly says that the Poincaré Lemma holds at the point $O \in \Omega$ in degree $q(1 \leq q \leq n)$ for the differential complex (1.9) if the following is true:

To each open neighborhood $U \subset \Omega$ of $O$ there is an open neighborhood $V \subset U$ of $O$ that has the following property:

$(\star)_{q}$

To every form $f \in \mathcal{C}^{\infty}\left(U ; \Lambda^{0, q}\right)$ such that $L f \equiv 0$ in $U$, there is a form $u \in \mathcal{C}^{\infty}\left(V ; \Lambda^{0, q-1}\right)$ such that $L u=f$ in $V$.

By the isomorphism indicated above, Property $(\star)_{q}$ is equivalent to the property that to every closed form $f \in \mathcal{C}^{\infty}\left(U ; \Lambda^{1, q}\right)$ there is a form $u \in \mathcal{C}^{\infty}\left(V ; \Lambda^{1, q-1}\right)$ such that $d u=f$ in $V$. Also notice that $(1.12)_{q}$ entails

To each open neighborhood $U \subset \Omega$ of $O$ there is an open neighborhood $V \subset U$ of $O$ that has the following property:

To every form $f \in \mathcal{C}^{\infty}\left(U ; \Lambda^{0, q}\right)$ such that $L f \equiv 0$ in $U$, there is a current $u \in \mathcal{D}^{\prime}\left(V ; \Lambda^{0, q-1}\right)$ such that $L u=f$ in $V$.

Now denote by $\widetilde{H}_{*}(\ldots)$ the reduced singular homology with complex coefficients. Outside of the trivial case $\Phi \equiv 0$ the codimension of a generic fibre $\mathcal{F}\left(z_{0}, U\right)$ is equal to two, i.e., $\operatorname{dim} \mathcal{F}\left(z_{0}, U\right)=n-1$ [of course, the dimension of a nongeneric fibre $\mathcal{F}\left(z_{0}, U\right)$ can be equal to any number between 0 and $n$, as when $\Phi=t_{1}^{2}+\cdots+t_{\nu}^{2}$ or $\left.\Phi=x t_{1}\right]$. We shall say that the $\mathcal{V}$-fibration of $\mathcal{M}$ is homologically trivial in degree $q(0 \leq q \leq n-1)$ at the point $O \in \Omega$ if the following is true:

To each open neighborhood $U$ of $O$ in $\Omega$ there is an open neighborhood $V \subset U$ of $O$ such that the following property holds:

$\forall z_{0} \in \mathbb{C}$, the inclusion $\mathcal{F}\left(z_{0}, V\right) \subset \mathcal{F}\left(z_{0}, U\right)$ induces the zero map $\widetilde{H}_{q}\left(\mathcal{F}\left(z_{0}, V\right)\right) \rightarrow \widetilde{H}_{q}\left(\mathcal{F}\left(z_{0}, U\right)\right)$.

The vanishing of the map $\widetilde{H}_{q}\left(\mathcal{F}\left(z_{0}, V\right)\right) \rightarrow \widetilde{H}_{q}\left(\mathcal{F}\left(z_{0}, U\right)\right)$ has the following meaning: when $q=0: \mathcal{F}\left(z_{0}, V\right)$ is contained in a single connected component of $\mathcal{F}\left(z_{0}, U\right)$; 
when $q \geq 1$ : every $q$-cycle in $\mathcal{F}\left(z_{0}, V\right)$ bounds in $\mathcal{F}\left(z_{0}, U\right)$;

when $q=n-1$ and $n \geq 2$ : no connected component of $\mathcal{F}\left(z_{0}, V\right)$ is compact.

[When $q=n-1$, the presence of the larger neighborhood $U$ is irrelevant.]

It should be pointed out that the validity of Property $(\star \star)_{q}$ for regular fibres $\mathcal{F}\left(z_{0}, U\right)$ [i.e., $z_{0}$ is a noncritical value of $Z$ or, equivalently, $y_{0}$ is not a critical value of $\left.t \rightarrow \Phi\left(x_{0}, t\right)\right]$ implies its validity for all fibres. The proofs of all the results stated below make use only of the validity of $(\star \star)_{q}$ for regular fibres $\mathcal{F}\left(z_{0}, U\right)$.

Under the hypotheses of the present work, i.e., $Z \in \mathcal{C}^{\omega}$, Treves [19] conjectured, and proved in the case $q=1$, the following

Theorem 1.1. For $\left(1.12^{\prime}\right)_{q}$ to be valid it is necessary that the $\mathcal{V}$-fibration of $\mathcal{M}$ be homologically trivial in degree $q-1$ at $O$. Conversely, if the latter is true, then the Poincaré Lemma holds at $O \in \Omega$, in degree $q$, for the differential complex (1.9).

In other words, the following entailments are true:

$$
\left(1.12^{\prime}\right)_{q} \Rightarrow(1.13)_{q-1} \Rightarrow(1.12)_{q}\left[\Rightarrow\left(1.12^{\prime}\right)_{q}\right]
$$

Actually, in the case $q=1$ a slightly weaker version of Theorem 1.1 was proved in [19]: to establish Property $(1.12)_{1}$ the validity of $(1.13)_{0}$ was needed not only at $O$ but at every point in a full neighborhood of $O$.

In full generality, i.e., under the sole hypothesis $Z \in \mathcal{C}^{\infty}(\Omega)$, Cordaro and Treves [5] established that $\left(1.12^{\prime}\right)_{q} \Rightarrow(1.13)_{q-1}$ for all $q=1, \ldots, n$.

In the present paper we limit our attention to the entailment $(1.13)_{q-1} \Rightarrow(1.12)_{q}$. For $q=1$ it was proved by Mendoza and Treves in [14] in full generality, i.e., under the hypothesis $Z \in \mathcal{C}^{\infty}$. For $q=n$ and $Z \in \mathcal{C}^{\omega}$ the same result was proved by Cordaro and Hounie [4].

Concerning this background two more remarks are in order:

1. When $n=1$ Property $(1.13)_{1}$ coincides with the solvability condition $(\mathcal{P})([15])$, as it should, since $(\mathcal{P}) \Leftrightarrow(1.12)_{1} \Leftrightarrow\left(1.12^{\prime}\right)_{1}$ when $n=1$. [That $(\mathcal{P}) \Rightarrow(1.12)_{1}$ is a particular case of $[10$, Theorem 7.3]. A fully general proof of the fact that $\left(1.12^{\prime}\right)_{1} \Rightarrow(\mathcal{P})$ was first given by Hörmander [11] and follows from an argument of R. Moyer. For a locally integrable vector field $L$ both proofs can be simplified, as shown in Sections VIII.8, VIII.9, VIII.10 of [20].] If $n=1$, one is dealing with a single vector field with smooth coefficients, $L=\partial / \partial t-\left(Z_{t} / Z_{x}\right) \partial / \partial x$. The real part of the symbol of $Z_{x} L, \Phi_{t} \xi-\Phi_{x} \tau+\iota \tau$, is equal to $\Phi_{t} \xi$ on any null bicharacteristic of the imaginary part. Condition $(\mathcal{P})$ states that, for each fixed $x$, the function $t \rightarrow \Phi_{t}(x, t)$ does not change sign, which is the same as saying that the level sets of its primitive, $t \rightarrow \Phi(x, t)$, are connected.

2. The work [6] formulates a hyperfunction version of Property $(1.12)_{q}$, and proves that it implies $(1.13)_{q-1}$, when $Z \in \mathcal{C}^{\infty}$ (generalizing the obvious hyperfunction version when the coefficients of the vector fields $L_{j}$ are analytic). The converse entailment is established in [7]. In other words, the hyperfunction version of Theorem 1.1 has been proved. What we still don't know is whether $(1.13)_{q-1} \Rightarrow(1.12)_{q}$ [or even $\left.(1.13)_{q-1} \Rightarrow\left(1.12^{\prime}\right)_{q}\right]$ when $q \geq 2$ and $Z$ is smooth but not analytic.

The present work is devoted to the proof that $(1.13)_{q-1} \Rightarrow(1.12)_{q}$ for $1 \leq q \leq n$ and $Z \in \mathcal{C}^{\omega}$, thus completing the proof of Theorem 1.1 when $Z \in \mathcal{C}^{\omega}$. The proof will rely on the following consequence of the Approximate Poincaré Lemma [20, Corollaries II.6.4 and II.6.5]: 
Lemma 1.2. To every open neighborhood $U$ of $O$ in $\Omega$ there is an open neighborhood $V \subset U$ of $O$ such that the following is true:

For any $z_{0} \in Z(V)$, the integral of any $L$-closed form $f \in$ $\mathcal{C}^{\infty}\left(U ; \Lambda^{0, q}\right)$ over any Lipschitz q-cycle $\gamma \subset \mathcal{F}\left(z_{0}, V\right)$ vanishes.

Property $(1.14)_{q}$ will allow us to apply De Rham's theorem and assert that the pullback to any $\mathcal{C}^{\infty}$ submanifold $\Lambda \subset \mathcal{F}\left(z_{0}, V\right)$ of any $L$-closed form $f \in \mathcal{C}^{\infty}\left(U ; \Lambda^{0, q}\right)$ is exact [for the standard exterior derivative on $\Lambda$; see $(2.3)_{q}$ ].

\section{The lift to the sphere. Two lemmas. Proof of the theorem}

Thanks to Lemma 1.2 we can select an interval $\mathcal{I}$ in the $x$-line, centered at zero, and a ball $r \mathbf{B}^{n}\left(\mathbf{B}^{n}\right.$ : open unit ball in $\left.\mathbb{R}^{n} ; r>0\right)$ such that $(1.14)_{q}$ is valid with $V=\mathcal{I} \times r \mathbf{B}^{n}(\subset U \subset \Omega)$. Rescaling allows us to assume $r>1$.

It is convenient (but not essential) to deal with compact fibres and for this to lift the analysis from $\mathcal{I} \times \mathbb{R}^{n}$ to $\mathcal{I} \times \mathbf{S}^{n}\left(\mathbf{S}^{n}\right.$ : unit sphere in $\left.\mathbb{R}^{n+1}\right)$. Call $\left(t_{1}, \ldots, t_{n}, t_{n+1}\right)$ the variable in $\mathbb{R}^{n+1}$, and call $\pi$ the coordinate projection $\left(x, t_{1}, \ldots, t_{n}, t_{n+1}\right) \rightarrow$ $\left(x, t_{1}, \ldots, t_{n}\right)$; it maps $\mathcal{I} \times \mathbf{S}^{n}$ onto $\mathcal{I} \times \overline{\mathbf{B}}^{n}\left(\overline{\mathbf{B}}^{n}:\right.$ closed unit ball in $\left.\mathbb{R}^{n}\right)$. We pull back to $\mathcal{I} \times \mathbf{S}^{n}$ the integrable structure on $\Omega$ defined by the function $Z$. Call $Z^{\pi}=x+\iota \Phi^{\pi}(x, t)$ the pullback $Z \circ \pi ; \Phi^{\pi}(x, t)$ is simply the restriction to $\mathcal{I} \times \mathbf{S}^{n}$ of $\Phi(x, t)$ regarded as a function independent of $t_{n+1}$. We have $\Phi^{\pi}(x, 0) \equiv 0$.

We denote by $\mathcal{C}^{\infty}\left(\mathcal{I} \times \mathbf{S}^{n} ; \Lambda^{0, q}\right)$ the space of differential $q$-forms on $\mathcal{I} \times \mathbf{S}^{n}$,

$$
f=\sum_{|J|=q} f_{J}(x, t) d t_{J}
$$

where $f_{J} \in \mathcal{C}^{\infty}\left(\mathcal{I} \times \mathbf{S}^{n}\right)$. As usual, $J=\left\{j_{1}, \ldots, j_{q}\right\}, 1 \leq j_{1}<\cdots<j_{q} \leq n+1$; of course, in $\mathbf{S}^{n}$ the variables $t_{j}$ are not independent: $t_{1}^{2}+\cdots+t_{n}^{2}+t_{n+1}^{2}=1$. We call $\mathcal{C}^{\infty}\left(\mathcal{I} \times \mathbf{S}^{n} ; \Lambda^{1, q}\right)$ the space of $(q+1)$-forms $d Z^{\pi} \wedge f$ with $f$ as in (2.1). The coordinate projection $\pi: \mathbf{S}^{n} \rightarrow \overline{\mathbf{B}}^{n}$ defines the pullback map $\pi^{*}: \mathcal{C}^{\infty}\left(\mathcal{I} \times \overline{\mathbf{B}}^{n} ; \Lambda^{j, q}\right) \rightarrow$ $\mathcal{C}^{\infty}\left(\mathcal{I} \times \mathbf{S}^{n} ; \Lambda^{j, q}\right)(j=0,1)$. The restriction of $\pi$ to $\mathbf{S}_{+}^{n}=\left\{\left(t_{1}, \ldots, t_{n+1}\right) \in\right.$ $\left.\mathbf{S}^{n} ; t_{n+1}>0\right\}$ defines an isomorphism $\mathcal{C}^{\infty}\left(\mathcal{I} \times \mathbf{B}^{n} ; \Lambda^{j, q}\right) \rightarrow \mathcal{C}^{\infty}\left(\mathcal{I} \times \mathbf{S}_{+}^{n} ; \Lambda^{j, q}\right)$. We may as well introduce the analogue of $L$ [see (1.8)]:

$$
f \rightarrow L^{\pi} f=d_{t} f-\iota d_{t} \Phi^{\pi} \wedge L_{0}^{\pi} f,
$$

with $L_{0}^{\pi}=\left(1+\iota \Phi_{x}^{\pi}\right)^{-1} \partial / \partial x$ acting on the coefficients of differential forms. We get the differential complexes (for $j=0,1$ ):

$$
L^{\pi}: \mathcal{C}^{\infty}\left(\mathcal{I} \times \mathbf{S}^{n} ; \Lambda^{j, q-1}\right) \rightarrow \mathcal{C}^{\infty}\left(\mathcal{I} \times \mathbf{S}^{n} ; \Lambda^{j, q}\right), \quad q=1,2, \ldots
$$

Now let $\mathcal{F}^{\pi}\left(z_{0}\right)$ be a fibre of the function $Z^{\pi}: \mathcal{I} \times \mathbf{S}^{n} \rightarrow \mathbb{C} ; \mathcal{F}^{\pi}\left(z_{0}\right)$ is defined by the equations $x=x_{0}, \Phi^{\pi}(x, t)=y_{0} ; \pi\left(\mathcal{F}^{\pi}\left(z_{0}\right)\right)=\mathcal{F}\left(z_{0}, \overline{\mathbf{B}}^{n}\right)$. Consider an arbitrary $L$-closed form $f \in \mathcal{C}^{\infty}\left(U ; \Lambda^{0, q}\right)$. If $\mathfrak{X}$ is any $\mathcal{C}^{\infty}$ submanifold of $\mathbf{S}^{n}$ contained in $\mathcal{F}^{\pi}\left(z_{0}\right)$ and if $\gamma$ is a smooth $q$-cycle in $\mathfrak{X}$, then $\pi(\gamma)$ is a smooth $q$-cycle contained in $\pi(\mathfrak{X}) \subset \mathcal{F}\left(z_{0}, \overline{\mathbf{B}}^{n}\right)$. By $(1.14)_{q}$ we have $\int_{\gamma} \pi^{*} f=\int_{\pi(\gamma)} f=0$ and De Rham's theorem leads to the following conclusion:

Given any $L$-closed form $f \in \mathcal{C}^{\infty}\left(U ; \Lambda^{0, q}\right)$, the pullback of $\pi^{*} f$ to any $\mathcal{C}^{\infty}$ submanifold $\mathfrak{X}$ of any fibre $\mathcal{F}^{\pi}\left(z_{0}\right)$ is exact. 
Since $L_{0}$ and $L$ commute we may apply $(2.3)_{q}$ with $L_{0}^{k} f$ in the place of $f$ :

Given any L-closed form $f \in \mathcal{C}^{\infty}\left(U ; \Lambda^{0, q}\right)$, the pullback of $\pi^{*}\left(L_{0}^{k} f\right)=\left(L_{0}^{\pi}\right)^{k} \pi^{*} f\left(k \in \mathbb{Z}_{+}\right)$to any $\mathcal{C}^{\infty}$ submanifold $\mathfrak{X}$ of a fibre $\mathcal{F}^{\pi}\left(z_{0}\right)$ is exact.

Let $p$ be an integer, $0 \leq p \leq n$, and let $\Omega$ be an open subset of $\mathcal{I} \times \mathbf{S}^{n}$. We shall denote by $\mathcal{C}_{*}^{\infty}\left(\Omega ; \Lambda^{0, p}\right)$ the subspace of $\mathcal{C}^{\infty}\left(\Omega ; \Lambda^{0, p}\right)$ consisting of the forms $F$ that vanish to infinite order on the intersection of $\Omega$ with every singular fibre of $Z^{\pi}$. By a singular fibre $\mathcal{F}^{\pi}\left(z_{0}\right)$ we mean a fibre of $Z^{\pi}$ such that $d_{t} \Phi^{\pi}\left(x_{0}, t\right)=0$ for some, but not necessarily all, $t \in \mathcal{F}^{\pi}\left(z_{0}\right)$; in other words, $z_{0}$ is a critical value of $Z^{\pi}$. Here and throughout the sequel we use the following terminology: A $\mathcal{C}^{\infty}$ function $f$ in an open subset $\mathfrak{Y}$ of a $\mathcal{C}^{\infty}$ manifold $\mathfrak{X}$ is said to vanish to infinite order on a set $E \subset \mathrm{Cl} \mathfrak{Y}$ if, given any differential operator $D$ with $\mathcal{C}^{\infty}$ coefficients in $\mathfrak{X}, D f(p) \rightarrow 0$ as the point $p \in \mathfrak{Y}$ approaches $E$. A $\mathcal{C}^{\infty}$ differential form in $\mathfrak{Y}$ is said to vanish to infinite order on $E$ if every one of its coefficients does.

Theorem 1.1 will be a consequence of the following two lemmas:

Lemma 2.1. Suppose that the form $f \in \mathcal{C}^{\infty}\left(\mathcal{I} \times \mathbf{S}^{n} ; \Lambda^{0, p}\right)$ is $L^{\pi}$-closed and that for every $k=0,1, \ldots$, the pullback of $\left(L_{0}^{\pi}\right)^{k} f$ to any $\mathcal{C}^{\infty}$ submanifold of any fibre of $Z^{\pi}$ is exact. Then there are a rectangle $\mathcal{R}=\{z=x+\iota y \in \mathbb{C} ;|x|<a,|y|<b\}(a, b>0)$ and a form $u_{0} \in \mathcal{C}^{\infty}\left(U^{\pi} ; \Lambda^{0, p-1}\right)$ such that $F=f-L^{\pi} u_{0} \in \mathcal{C}_{*}^{\infty}\left(U^{\pi} ; \Lambda^{0, p}\right)$, where $U^{\pi}=\left(Z^{\pi}\right)^{-1}(\mathcal{R})$.

Lemma 2.2. Let $\mathcal{R}$ and $U^{\pi}$ be as in Lemma 2.1; suppose the pullback of $F \in$ $\mathcal{C}_{*}^{\infty}\left(U^{\pi} ; \Lambda^{0, p}\right)$ to any $\mathcal{C}^{\infty}$ submanifold of any fibre of $Z^{\pi}$ in $U^{\pi}$ is exact. Then, possibly after contracting $\mathcal{R}$ about zero, there is $v \in \mathcal{C}_{*}^{\infty}\left(U^{\pi} ; \Lambda^{0, p-1}\right)$ such that $\left(F-d_{t} v\right) \wedge d_{t} \Phi^{\pi}=0$.

The proof of Lemma 2.1 is given in Section 3, and that of Lemma 2.2 in Sections 4 and 5. Here we show how Lemmas 2.1 and 2.2 entail Theorem 1.1. Actually we need the following consequence of those lemmas:

Lemma 2.3. If $f \in \mathcal{C}^{\infty}\left(\mathcal{I} \times \mathbf{S}^{n} ; \Lambda^{0, p}\right)$ satisfies the hypotheses of Lemma 2.1 and if the sets $\mathcal{R}, U^{\pi}$ are as in Lemma 2.2 , then there are forms $w \in \mathcal{C}^{\infty}\left(U^{\pi} ; \Lambda^{0, p-1}\right)$ and $F_{1} \in \mathcal{C}_{*}^{\infty}\left(U^{\pi} ; \Lambda^{0, p-1}\right)$ such that

$$
f=L^{\pi} w+d_{t} \Phi^{\pi} \wedge F_{1} \quad \text { in } U^{\pi} .
$$

Proof of Lemma 2.3. Let the forms $f, u_{0}$ and $F$ be as in Lemma 2.1, and let $v$ be related to $F$ as in Lemma 2.2. In the open set $U_{0}^{\pi} \subset U^{\pi}$ where $d_{t} \Phi^{\pi} \neq 0, \Phi^{\pi}$ may be used as one of the coordinates; and $\left(F-d_{t} v\right) \wedge d_{t} \Phi^{\pi}=0 \Rightarrow \exists F_{2} \in \mathcal{C}^{\infty}\left(U_{0}^{\pi} ; \Lambda^{0, p-1}\right)$ such that

$$
F-d_{t} v=d_{t} \Phi^{\pi} \wedge F_{2} \quad \text { in } U_{0}^{\pi} .
$$

When $p=1, F_{2}$ is a uniquely determined function; $F_{2}$ belongs to $\mathcal{C}_{*}^{\infty}\left(U^{\pi}\right)$ since the coefficients of $F-d_{t} v$ do. When $p \geq 2$ the form $F_{2}$ is not unique since we can add to it any form $R \wedge d_{t} \Phi^{\pi}, R \in \mathcal{C}^{\infty}\left(U^{\pi} ; \Lambda^{0, p-2}\right)$. We may, however, define $F_{2}$ unambiguously by requiring that its contraction with the gradient $\nabla_{t} \Phi^{\pi}$ vanish identically. Consider an arbitrary point $\left(x_{0}, t_{0}\right)$ of $U_{0}^{\pi}$ and provisionally freeze $x$ at $x_{0}$. In a suitably small open neighborhood of $t_{0}$ we select an orthonormal basis $X_{1}, \ldots, X_{n}$ of vectors tangent to the sphere $\mathbf{S}^{n}$ with $X_{n}=\nabla_{t} \Phi^{\pi}\left(x_{0}, t\right) /\left\|\nabla_{t} \Phi^{\pi}\left(x_{0}, t\right)\right\|$; this entails

$$
\left\langle d_{t} \Phi^{\pi}, X_{i}\right\rangle=0 \quad \text { if } i<n, \quad\left\langle d_{t} \Phi^{\pi}, X_{n}\right\rangle=\left\|\nabla_{t} \Phi^{\pi}\left(x_{0}, t\right)\right\| .
$$


Given any set of $p-1$ integers $i_{\alpha}, 1 \leq i_{1}<\cdots<i_{p-1}<n$, we derive from (2.6):

$$
\left\langle F-d_{t} v, X_{i_{1}} \wedge \cdots \wedge X_{i_{p-1}} \wedge X_{n}\right\rangle= \pm\left\|\nabla_{t} \Phi^{\pi}\left(x_{0}, t\right)\right\|\left\langle F_{2}, X_{i_{1}} \wedge \cdots \wedge X_{i_{p-1}}\right\rangle
$$

which shows that the coefficients of $F_{2}$ and their derivatives can be estimated uniformly in terms of the corresponding quantities for $F-d_{t} v$ divided by $\left\|\nabla_{t} \Phi^{\pi}\left(x_{0}, t\right)\right\|$. Thanks to a Lojasiewicz type inequality (see Lemma 4.1) and to the fact that the coefficients of $F-d_{t} v$ vanish to infinite order on any level set of $\Phi^{\pi}$ containing a point where $\nabla_{t} \Phi^{\pi}=0$ we conclude that $F_{2} \in \mathcal{C}_{*}^{\infty}\left(U^{\pi} ; \Lambda^{0, p-1}\right)$. We see that (2.5) is valid with $w=u_{0}+v$ and $F_{1}=F_{2}+\iota L_{0}^{\pi} v \in \mathcal{C}_{*}^{\infty}\left(U^{\pi} ; \Lambda^{0, p-1}\right)$.

Next we show how Theorem 1.1 follows from the preceding lemmas. We deal with a form $f \in \mathcal{C}^{\infty}\left(\mathcal{I} \times \mathbf{S}^{n} ; \Lambda^{q}\right)$ which satisfies the hypotheses of Lemma 2.1 (for $p=q)$. It is convenient to introduce the differential form $\lambda^{\pi}=-\iota\left(1+\iota \Phi_{x}^{\pi}\right)^{-1} d_{t} \Phi^{\pi}$. If $\psi \in \mathcal{C}^{\infty}\left(\mathcal{I} \times \mathbf{S}^{n}\right)$, then $L^{\pi} \psi=d_{t} \psi+\psi_{x} \lambda^{\pi}$; the condition $L^{\pi} \circ L^{\pi}=0$ is equivalent to

$$
L^{\pi} \lambda^{\pi}=0
$$

Let $w$ and $F_{1}$ be as in $(2.5)$. Set $g=\iota\left(1+\iota \Phi_{x}^{\pi}\right) F_{1} \in \mathcal{C}_{*}^{\infty}\left(U^{\pi} ; \Lambda^{0, q-1}\right)$; we rewrite $(2.5)$ as

$$
f-L^{\pi} w=\lambda^{\pi} \wedge g
$$

From the equation $L^{\pi} f=0$ and from (2.7) we deduce that $\lambda^{\pi} \wedge L^{\pi} g=\lambda^{\pi} \wedge d_{t} g=0$, which is equivalent to $d_{t} \Phi^{\pi} \wedge d_{t} g=0$. Taking into account the fact that $g$ vanishes to infinite order on every singular fibre of $Z^{\pi}$ we conclude that the pullback of $g$ to any smooth submanifold of any fibre of $Z^{\pi}$ contained in $U^{\pi}$ is closed.

It is at this juncture that we avail ourselves of Hypothesis $(1.13)_{q-1}$. We use the map $\pi$ to project the open "Northern" hemisphere $\mathbf{S}_{+}^{n}$ onto the open unit ball $\mathbf{B}^{n}$ and the intersection $U^{\pi} \cap\left(\mathcal{I} \times \mathbf{S}_{+}^{n}\right)$ onto an open neighborhood $U^{\prime}$ of 0 in $\mathcal{I} \times \mathbb{R}^{n}$. By virtue of $(1.13)_{q-1}$ there is an open interval $\mathcal{I}^{\prime} \subset \mathcal{I}$ centered at zero and a number $r^{\prime}, 0<r^{\prime} \leq 1$ (independent of $f, g$, etc.), such that $\mathcal{I}^{\prime} \times r^{\prime} \mathbf{B}^{n} \subset U^{\prime}$ and that, given any $z_{0} \in \mathbb{C}$ such that $\mathcal{F}\left(z_{0}, \mathcal{I}^{\prime} \times r^{\prime} \mathbf{B}^{n}\right) \neq \varnothing$, the following is true: if $q=1, \mathcal{F}\left(z_{0}, \mathcal{I}^{\prime} \times r^{\prime} \mathbf{B}^{n}\right)$ is contained in a single connected component of the fibre $\mathcal{F}\left(z_{0}, \mathcal{I} \times \mathbf{B}^{n}\right)$; if $q \geq 2$ the pullback of $\pi_{*} g$ to any $\mathcal{C}^{\infty}$ submanifold of $\mathcal{F}\left(z_{0}, \mathcal{I}^{\prime} \times r^{\prime} \mathbf{B}^{n}\right)$ is exact.

Case $q=1$. Here it suffices to note that the function $\pi_{*} g$ must be constant on each fibre $\mathcal{F}\left(z_{0}, \mathcal{I}^{\prime} \times r^{\prime} \mathbf{B}^{n}\right)$. Thus there is an open neighborhood $U^{\prime \pi} \subset U^{\pi}$ of the point $(0, \ldots, 0,1)$ in $\mathcal{I} \times \mathbf{S}^{n}$ in which $g(x, t)=\tilde{g}\left(x, \Phi^{\pi}(x, t)\right)$, with $\tilde{g}$ a $\mathcal{C}^{\infty}$ function in an open disk $\Delta \subset \mathbb{C}$ centered at 0 (again thanks to the fact that $g$ vanishes to infinite order on the singular fibres of $\left.Z^{\pi}\right)$. Solve the Cauchy-Riemann equation $\partial \tilde{\chi} / \partial \bar{z}=-\frac{1}{2} \tilde{g}$ and set $\chi(x, t)=\tilde{\chi}\left(x, \Phi^{\pi}(x, t)\right)$; then

$$
L^{\pi} \chi=\left[(\partial \tilde{\chi} / \partial \bar{z}) \circ Z^{\pi}\right] L \bar{Z}^{\pi}=2 \lambda^{\pi}\left[(\partial \tilde{\chi} / \partial \bar{z}) \circ Z^{\pi}\right]=-\lambda^{\pi} g .
$$

Case $q \geq 2$. Call $G$ the pullback of $\pi_{*} g$ to $\mathcal{I}^{\prime} \times \mathbf{S}^{n}$ under the map $\pi^{\prime}$ : $\left(x, t_{1}, \ldots, t_{n}, t_{n+1}\right) \rightarrow\left(x, r^{\prime} t_{1}, \ldots, r^{\prime} t_{n}\right) ; G$ satisfies the same hypotheses as $F$ in Lemma 2.2, for $p=q-1$ and with $\mathcal{I}^{\prime}$ substituted for $\mathcal{I}$. Returning to the original product $\mathcal{I} \times \mathbf{S}^{n}$ we conclude that there are an open neighborhood $U^{\prime \pi} \subset U^{\pi}$ of $(0, \ldots, 0,1)$ in $\mathcal{I} \times \mathbf{S}^{n}$ and a form $\chi \in \mathcal{C}^{\infty}\left(U^{\prime \pi} ; \Lambda^{0, q-2}\right)$ such that $d_{t} \Phi^{\pi} \wedge\left(g-d_{t} \chi\right)=0$, i.e., $\lambda^{\pi} \wedge\left(g-d_{t} \chi\right)=0$ in $U^{\prime \pi}$. Since $L^{\pi}\left(\lambda^{\pi} \wedge \chi\right)=-\lambda^{\pi} \wedge L^{\pi} \chi=-\lambda^{\pi} \wedge d_{t} \chi$ by 
(2.7), the equation

$$
L^{\pi}\left(\lambda^{\pi} \wedge \chi\right)=-\lambda^{\pi} \wedge g
$$

is valid in $U^{\prime \pi}$ (for any $q, 1 \leq q \leq n$ ). Combining (2.8) and (2.9) yields

$$
f=L^{\pi}\left(w-\lambda^{\pi} \wedge \chi\right) \text {. }
$$

\section{Proof of Lemma 2.1}

We begin with a few remarks aimed at simplifying the situation and at laying the ground for the formal proofs.

There are two cases in which the Poincaré Lemma is known to hold at every point of $\Omega$ and in each degree $q=1, \ldots, n$ : the "essentially real case" $\Phi \equiv 0$, in which $\mathcal{V}$ is spanned by $\partial / \partial t_{1}, \ldots, \partial / \partial t_{n}$; and the "elliptic case", in which $d_{t} \Phi$ never vanishes and $\Phi$ can be chosen as one of the local coordinates. If $x, y=\Phi, t_{1}, \ldots, t_{n-1}$ form a complete coordinate system in a neighborhood of the origin, then $Z=x+\iota y$, and $\mathcal{V}$ is spanned over $U$ by the vector fields $\partial / \partial \bar{z}, \partial / \partial t_{1}, \ldots, \partial / \partial t_{n-1}$ ([20, end of Section VI.7]). We shall assume that none of these two extreme cases obtains: the singular set of the function $Z,\left\{(x, t) \in \Omega ; d_{t} \Phi(x, t)=0\right\}$, will be a proper analytic subset of $\Omega$ which contains the origin.

By a singular fibre of $Z^{\pi}$ we mean a fibre $\mathcal{F}^{\pi}\left(z_{0}\right)$ that intersects the singular set of $Z^{\pi}$; then $z_{0}$ is a critical value of $Z^{\pi}$. Off the equator, i.e., when $t_{n+1} \neq 0$, the projection $\pi$ is a bijection of the singular set of $Z^{\pi}$ onto that of $Z$ in $\mathcal{I} \times \mathbf{B}^{n}$. But $\Phi^{\pi}$ will have singular points on the equator (where $t_{1}^{2}+\cdots+t_{n}^{2}=1$ ), defined by the equations

$$
t_{j} \partial \Phi / \partial t_{k}=t_{k} \partial \Phi / \partial t_{j}, \quad 1 \leq j<k \leq n .
$$

The range of $Z^{\pi}: \mathcal{I} \times \mathbf{S}^{n} \rightarrow \mathbb{C}$ is the set $\{x+\iota y ; x \in \mathcal{I}, m(x) \leq y \leq M(x)\}$, where $m(x)=\operatorname{Min}_{t \in \mathbf{S}^{n}} \Phi^{\pi}(x, t), M(x)=\operatorname{Max}_{t \in \mathbf{S}^{n}} \Phi^{\pi}(x, t)$. The critical values of $Z^{\pi}$ form a subanalytic subset $\mathcal{S}^{\pi}$ of the range of $Z^{\pi}$. For fixed $x \in \mathcal{I}$ the analytic subvariety of $\mathbf{S}^{n}$ defined by the equation $d_{t} \Phi^{\pi}(x, t)=0$ has finitely many connected components, on each one of which $\Phi^{\pi}(x, t)=$ const. Thus the intersection of $\mathcal{S}^{\pi}$ with any vertical line $x=$ const. is a finite set, which means that $\mathcal{S}^{\pi}$ is a finite union of analytic curves $\ell_{j}=\left\{x+\iota y ; x \in \mathcal{I}_{j}, y=\psi_{j}(x)\right\}\left(j=1, \ldots, r ; \mathcal{I}_{j}\right.$ : open subinterval of $\mathcal{I})$ and of single points $\zeta_{k}(k=1, \ldots, s)$. The curves $\ell_{j}$ are pairwise disjoint; the points $\zeta_{k}$ are either isolated or lie on the boundaries of one or more curves $\ell_{j}$. In passing, note that, except in the trivial case $\Phi \equiv 0$, which we shall not consider, the points $x \in \mathcal{I}$ such that $d_{t} \Phi(x, t) \equiv 0$ for all $t$ are isolated. We select the rectangle $\mathcal{R}=\{x+\iota y ;|x|<a,|y|<b\}$ (Lemma 2.1) so small that: (i) if $|x|<a$ and if $d_{t} \Phi(x, t) \equiv 0$ for all $t$, then $x=0$; (ii) the only point $\zeta_{k}$ lying in $\mathcal{R}$ is the origin and the only curves $\ell_{j}$ intersecting $\mathcal{R}$ are those passing through the origin. By a good choice of $a$ and $b$ we can ensure that the curves $\ell_{j}$ only meet the boundary of $\mathcal{R}$ on its vertical sides, $y= \pm b$. It is also convenient to replace each curve $\ell_{j}$ which is analytic at 0 by its left and right halves. Thus we can subdivide the family of curves $\ell_{j}$ that intersect $\mathcal{R}$ into two subfamilies: those curves, called $\ell_{j}^{+}$from now on, defined by $0<x<a, y=\psi_{j}^{+}(x), j=1, \ldots, r^{+}$; and those, called $\ell_{k}^{-}$, defined by $-a<x<0, y=\psi_{k}^{-}(x), k=1, \ldots, r^{-}$. After relabeling we have $-b<\psi_{1}^{ \pm}<\cdots<\psi_{r^{ \pm}}^{ \pm}<b$. Keep in mind that $\psi_{j}^{+}(0)=\psi_{k}^{-}(0)=0, j=1, \ldots, r^{+}$, $k=1, \ldots, r^{-}$. 
The preimage under the map $Z^{\pi}$ of $\mathcal{S}^{\pi} \cap \mathcal{R}$ is a subanalytic set $\mathcal{A}$. We shall call $\mathcal{A}^{+}$(resp., $\mathcal{A}^{-}$) the part of $\mathcal{A}$ on which $x>0$ (resp., $x<0$ ); $\mathcal{A}=\mathcal{A}^{+} \cup \mathcal{A}^{-} \cup \mathcal{F}^{\pi}(0)$. Theorem 9.2 in [17] will enable us to complete the proof of Lemma 2.1. We describe now how we shall apply that result. We regard $\mathcal{A}$ as a fibre bundle over $(-a, a)$ whose fibre at $x$ is the union $\mathcal{A}_{x}$ of the fibres $\mathcal{F}^{\pi}(x+\iota y), x+\iota y \in \mathcal{S}^{\pi} \cap \mathcal{R}$. Sussman's theorem states that there is a stratification of $\mathcal{A}$ and one of $(-a, a)$ such that the fibre bundle $\mathcal{A}$ can be subanalytically trivialized over any stratum of $(-a, a)$. The meaning of the latter is as follows. The base projection $(x, t) \rightarrow x$ maps an arbitrary stratum $\Sigma$ of $\mathcal{A}$ onto one, $S_{\Sigma}$, of $(-a, a)$; there are an analytic submanifold $\mathfrak{M}_{0}$ of $\mathbf{S}^{n}$ and a subanalytic isomorphism $\tau_{\Sigma}: S_{\Sigma} \times \mathfrak{M}_{0} \rightarrow \Sigma$ which maps $\{x\} \times \mathfrak{M}_{0}$ onto $\Sigma_{x}=\Sigma \cap \mathcal{A}_{x}$ for each $x \in S_{\Sigma}$. The map $\tau_{\Sigma}$ is an analytic diffeomorphism; moreover, both $\tau_{\Sigma}$ and its inverse are subanalytic in $(-a, a) \times \mathbf{S}^{n}$ : as we approach the boundary points of $S_{\Sigma} \times \mathfrak{M}_{0}$ they behave "subanalytically". In particular, their derivatives cannot "blow up" faster than some negative power of the distance to the boundary.

Any stratification of an interval consists of finitely many points and of finitely many open intervals. After decreasing $a>0$ we may, and shall, assume that the only strata of $(-a, a)$ are $(0, a),\{0\}$ and $(-a, 0)$.

We now proceed with the formal proofs; throughout the sequel $p$ will be an integer, $1 \leq p \leq n$.

Our first concern will be with the fibre of $Z^{\pi}$ at $0, \mathcal{F}^{\pi}(0)$. Obviously $\mathcal{F}^{\pi}(0) \subset U^{\pi}$ (see Lemma 2.1). To say that $\mathcal{F}^{\pi}(0)=\{0\} \times \mathbf{S}^{n}$ is the same as saying that $\Phi(x, t)=$ $x \Phi_{1}(x, t)$ with $\Phi_{1} \in \mathcal{C}^{\omega}\left(\mathcal{I} \times r \mathbf{B}^{n}\right)$ (keep in mind that $r>1$ ), i.e., $Z=x\left(1+\iota \Phi_{1}\right)$. Recall that $Z_{x}(x, 0)=1$, hence $\Phi_{1}(x, 0)=0$ for all $x \in \mathcal{I}$. Possibly after rescaling we may write $1+\iota \Phi_{1}=e^{\Psi}, \Psi \in \mathcal{C}^{\omega}\left(\mathcal{I} \times r \mathbf{B}^{n}\right)$. Thus $Z(x, t)=x e^{\Psi(x, t)}$. On the other hand we have $L=d_{t}-Z_{x}^{-1} d_{t} Z \wedge \partial_{x}$; and since $d_{t} Z=Z d_{t} \Psi$, we have

$$
L=d_{t}+Z \mu \wedge \partial_{x}, \quad \mu=-\left(1+\iota \Phi_{x}\right)^{-1} d_{t} \Psi .
$$

Note also that if $f \in \mathcal{C}^{\infty}\left(\mathcal{I} \times r \mathbf{B}^{n} ; \Lambda^{0, p}\right)$, then $\left.L f\right|_{x=0}=\left.d_{t} f\right|_{x=0}$.

Lemma 3.1. Suppose $\mathcal{F}^{\pi}(0)=\{0\} \times \mathbf{S}^{n}$ and $f \in \mathcal{C}^{\infty}\left(\mathcal{I} \times r \mathbf{B}^{n} ; \Lambda^{0, p}\right)$ is such that $L f$ vanishes to infinite order at $x=0$. Let $\omega$ be a $\mathcal{C}^{\infty}(p-1)$-form on $r \mathbf{B}^{n}$ such that $d_{t} \omega=\left.f\right|_{x=0}$. Then there is a form $u \in \mathcal{C}^{\infty}\left(\mathcal{I} \times r \mathbf{B}^{n} ; \Lambda^{0, p-1}\right)$ such that $L u-f$ vanishes to infinite order at $x=0$ and $\left.u\right|_{x=0}=\omega$.

Moreover, if $\tilde{u} \in \mathcal{C}^{\infty}\left(\mathcal{I} \times r \mathbf{B}^{n} ; \Lambda^{0, p-1}\right)$ is another form with the same properties, the following can be said. When $p \geq 2$, there is $v \in \mathcal{C}^{\infty}\left(\mathcal{I} \times r \mathbf{B}^{n} ; \Lambda^{0, p-2}\right)$ such that $u-\tilde{u}-L v$ vanishes to infinite order at $x=0$ and $\left.v\right|_{x=0} \equiv 0$. When $p=1$, the Taylor expansion of $u-\tilde{u}$ with respect to $x$, about $x=0$, is equal to a formal power series $\sum_{\nu=1}^{\infty} c_{\nu} Z^{\nu}$ with $c_{\nu} \in \mathbb{C}$ for all $\nu$.

Proof. Consider a differential form $f_{k}=Z^{k} g_{k}$ for some $k \in \mathbb{Z}_{+}$and some $g_{k} \in$ $\mathcal{C}^{\infty}\left(\mathcal{I} \times r \mathbf{B}^{n} ; \Lambda^{0, p}\right)$; if $L f_{k}=Z^{k} L g_{k}$ vanishes to infinite order at $x=0$, then so does $L g_{k}$. Putting $x=0$ in $L g_{k}$ yields $d_{t}\left(\left.g_{k}\right|_{x=0}\right)=0$, whence $\left.g_{k}\right|_{x=0}=d_{t} v_{k}$ for some $v_{k} \in \mathcal{C}^{\infty}\left(r \mathbf{B}^{n} ; \Lambda^{p-1}\right)$. We define

$$
f_{k+1}=f_{k}-L\left(Z^{k} v_{k}\right)=Z^{k}\left(g_{k}-d_{t} v_{k}\right)=Z^{k}\left(x h_{k}\right)=Z^{k+1}\left(e^{-\Psi} h_{k}\right)=Z^{k+1} g_{k+1} .
$$

Note that $L f_{k+1}=L f_{k}$ vanishes to infinite order at $x=0$. We can therefore repeat the preceding argument.

Starting with $f_{0}=f$ and $v_{0}=\omega$ this procedure produces a sequence of forms $v_{k} \in \mathcal{C}^{\infty}\left(\mathcal{I} \times r \mathbf{B}^{n} ; \Lambda^{0, p-1}\right)$. We select a function $\chi \in \mathcal{C}^{\infty}(\mathbb{R}), \chi(\tau)=1$ if $|\tau|<1$, 
$\chi(\tau)=0$ if $|\tau|>2$, and numbers $\epsilon_{\nu} \rightarrow+0$ such that the series $\sum_{\nu=0}^{\infty} \chi\left(x / \epsilon_{\nu}\right) Z^{\nu} v_{\nu}$ converges in the space $\mathcal{C}^{\infty}\left(\mathcal{I} \times r \mathbf{B}^{n} ; \Lambda^{0, p-1}\right)$ to a true differential form $u$. It is checked at once that $L u-f$ vanishes to infinite order at $x=0$; and of course $\left.u\right|_{x=0}=\omega$.

Now let $\tilde{u} \in \mathcal{C}^{\infty}\left(\mathcal{I} \times r \mathbf{B}^{n} ; \Lambda^{0, p-1}\right)$ have the same properties as $u$. If $p \geq 2$ we may apply the result just proved to $w=u-\tilde{u}$ since $\left.w\right|_{x=0}=0$ and $L w$ vanishes to infinite order at $x=0$.

Lastly, assume $p=1$. Write the Taylor expansion of $w$ with respect to $x$ about zero as $\sum_{\nu=1}^{\infty} x^{\nu} w_{\nu}(t)$. We solve the equation $Z(x, t)=Z$ with respect to $x$ : $x=x(Z, t)=\sum_{\nu=1}^{\infty} Z^{\nu} x_{\nu}(t)$, and substitute into $\sum_{\nu=1}^{\infty} x^{\nu} w_{\nu}(t)$. We end up with a formal series $\sum_{\nu=1}^{\infty} c_{\nu}(t) Z^{\nu}$. Since $L w$ vanishes to infinite order at $x=0$ we have (in the sense of formal power series) $\sum_{\nu=1}^{\infty} Z^{\nu} L c_{\nu}(t)=0$, which is only possible if $L c_{\nu}=d_{t} c_{\nu}=0$ for all $\nu$. Thus the Taylor expansion of $w$ with respect to $x$ is equal to $\sum_{\nu=1}^{\infty} c_{\nu} Z^{\nu}, c_{\nu} \in \mathbb{C}$.

We now focus on the case $\mathcal{F}^{\pi}(0) \neq\{0\} \times \mathbf{S}^{n}$. We are going to use a subanalytic stratification of $\mathcal{F}^{\pi}(0)$ [for instance the natural one, defined by the vanishing of the partial derivatives of the function $\left.\Phi^{\pi}(\cdot, 0)\right]$. We shall avail ourselves of the basic property of any subanalytic stratification, namely that the closure of any stratum is a union of strata (see, e.g., [17, Theorem 9.1]).

Let $\Sigma_{i}^{(\kappa)}\left(i=1, \ldots, l_{\kappa}\right)$ be the strata of $\mathcal{F}^{\pi}(0)$ of codimension $\kappa$. We denote by $\{0\} \times K$ a connected component of $\left(\mathrm{Cl}_{1}^{(\kappa)}\right) \cup \cdots \cup\left(\mathrm{Cl} \Sigma_{l_{\kappa}}^{(\kappa)}\right)$; here $K$ is a subset of $\mathbf{S}^{n}$. After relabeling we may assume that $\{0\} \times K=\left(\mathrm{Cl} \Sigma_{1}^{(\kappa)}\right) \cup \cdots \cup\left(\mathrm{Cl} \Sigma_{l}^{(\kappa)}\right)$ $\left(1 \leq l \leq l_{\kappa}\right)$. We define $\{0\} \times K^{0}=\Sigma_{1}^{(\kappa)} \cup \cdots \cup \Sigma_{l_{\kappa}}^{(\kappa)}$; since $j \neq k \Rightarrow \Sigma_{j}^{(\kappa)} \cap \Sigma_{k}^{(\kappa)}=\varnothing$, $K^{0}$ is a $\mathcal{C}^{\omega}$ submanifold of $\mathbf{S}^{n}$, it is the regular part of $K ; K=\mathrm{Cl} K^{0}$ and one may think of $K \backslash K^{0}$ as the boundary of $K^{0}$.

We make use of the standard Riemannian metric on $\mathbf{S}^{n}$ and of the associated norms on the tangent bundle $T \mathbf{S}^{n}$ and on the exterior algebra $\Lambda \mathbb{C} T^{*} \mathbf{S}^{n}$; and of a tubular neighborhood $\mathcal{T}$ of $K^{0}$ in $\mathbf{S}^{n}$ which "tapers off" at the boundary $K \backslash K^{0}$, as we now make precise. For each $t^{0} \in K^{0}$ let $\sigma_{t^{0}}$ denote the open geodesic $\kappa$-ball of radius $\delta\left(t^{0}\right)$ centered at $t^{0}$, whose tangent space at $t^{0}$ is equal to the normal space $N_{t^{0}} K^{0}$ to $K^{0}$ in $\mathbf{S}^{n}$ [the normal bundle of $K^{0}$ will be denoted by $N K^{0}$ ]. The function $K^{0} \ni t^{0} \rightarrow \delta\left(t^{0}\right) \in\left(0, \delta_{0}\right]$ is taken to be $\mathcal{C}^{\infty}$ and to tend to zero at $K \backslash K^{0}$. We define

$$
\mathcal{T}=\bigcup_{t^{0} \in K^{0}} \sigma_{t^{0}}
$$

We require that the exponential map induce a diffeomorphism onto $\mathcal{T}$ of the neighborhood of the zero section in $N K^{0}, \mathcal{T}_{1}=\left\{\left(t^{0}, \nu\right) \in N K^{0} ;|\nu|<\delta\left(t^{0}\right)\right\}$. Known properties of analytic sets ([12, pp. 121-129]; see also Exposés No. 21, 22, 23, in [16]) enable us to impose the following condition:

$$
\exists c, \gamma>0 \text { such that } \delta\left(t^{0}\right) \geq c\left[\operatorname{dist}\left(t^{0}, K \backslash K^{0}\right)\right]^{\gamma}, \quad \forall t^{0} \in K^{0} .
$$

It is not precluded that $K^{0}$ be closed (i.e., compact), in which case, of course, $\{0\} \times K^{0}$ is equal to a single stratum of $\mathcal{F}^{\pi}(0)$ and the validity of $(3.3)$ is obvious.

We coordinatize $\mathcal{T} \backslash K^{0}$ by "blowing up" the set $\mathcal{T}_{1} \subset N K^{0}$ at the zero section. Precisely, we introduce, as a "radial coordinate" in $\mathcal{T} \backslash K^{0}$, the geodesic distance $\rho(t)=\operatorname{dist}\left(t, t^{0}\right)$ between $t^{0} \in K^{0}$ and $t \in \sigma_{t^{0}}$. We call $\theta$ the fibre variable in the normal sphere bundle $\mathbf{S} N K^{0}$ and $\Theta$ the total variable in $\mathbf{S} N K^{0}: \Theta=\left(t^{0}, \theta\right)$. We 
shall make use of the manifold $\mathcal{T}_{2}=\left\{(\Theta, \rho) \in \mathbf{S} N K^{0} \times \mathbb{R}_{+} ; 0<\rho<\delta\left(t^{0}\right)\right\}$ and of the "natural" diffeomorphism of $\mathcal{T}_{2}$ onto $\mathcal{T} \backslash K^{0}$, obtained by composing

$$
\mathcal{T}_{2} \ni\left(\left(t^{0}, \theta\right), \rho\right) \rightarrow\left(t^{0}, \rho \theta\right) \in \mathcal{T}_{1} \backslash 0 \stackrel{\exp }{\rightarrow} \mathcal{T} \backslash K^{0} .
$$

The exterior algebra $\Lambda \mathbb{C} T^{*} \mathcal{T}$ gets the structure of a Hilbert vector bundle from the Riemannian structure of $\mathcal{T} \subset \mathbf{S}^{n}$. We use it to define, for each $q=0,1, \ldots, n$, a vector subbundle of $\Lambda^{q} \mathbb{C} T^{*}\left(\mathcal{T} \backslash K^{0}\right)$ :

$$
\begin{gathered}
\mathbf{E}^{(0)}=\Lambda^{0} \mathbb{C} T^{*}\left(\mathcal{T} \backslash K^{0}\right) \cong\left(\mathcal{T} \backslash K^{0}\right) \times \mathbb{C} ; \\
\text { if } q \geq 1, \quad \mathbf{E}^{(q)}=\left[\Lambda^{q} \mathbb{C} T^{*}\left(\mathcal{T} \backslash K^{0}\right)\right] \ominus\left[d \rho \wedge \Lambda^{q-1} \mathbb{C} T^{*}\left(\mathcal{T} \backslash K^{0}\right)\right] .
\end{gathered}
$$

Since $d \rho \wedge \mathbf{E}^{(q-1)}=d \rho \wedge \Lambda^{q-1} \mathbb{C} T^{*}\left(\mathcal{T} \backslash K^{0}\right)$ we have the orthogonal sum decomposition

$$
\Lambda^{q} \mathbb{C} T^{*}\left(\mathcal{T} \backslash K^{0}\right)=\mathbf{E}^{(q)} \oplus\left(d \rho \wedge \mathbf{E}^{(q-1)}\right) .
$$

Of course, $\left.\Lambda^{q} \mathbb{C} T^{*} \mathcal{T} \cong \Lambda^{0, q}\right|_{\mathcal{I} \times \mathcal{T}}$ by pullback under the coordinate map $(x, t) \rightarrow t$. This allows us to view $\mathbf{E}^{(q)}$ as a subbundle of $\Lambda^{0, q}$ over $\mathcal{I} \times\left(\mathcal{T} \backslash K^{0}\right)$. We can also pull back $\Lambda^{q} \mathbb{C} T^{*}\left(\mathbf{S} N K^{0}\right)$ to $\mathcal{I} \times \mathcal{T}_{2}$ via the coordinate map $(x, \Theta, \rho) \rightarrow \Theta$. The "blow up" map $\mathcal{T}_{2} \cong \mathcal{T} \backslash K^{0}$ induces a natural isomorphism of the vector bundles

$$
\Lambda^{q} \mathbb{C} T^{*}\left(\mathbf{S} N K^{0}\right) \cong \mathbf{E}^{(q)} .
$$

We may now introduce the differential operators

$$
L_{\rho}=\partial / \partial \rho-\iota\left(1+\iota \Phi_{x}^{\pi}\right)^{-1}\left(\partial \Phi^{\pi} / \partial \rho\right) \partial / \partial x
$$

acting on $\mathcal{C}^{\infty}\left(\mathcal{I} \times \mathcal{T}_{2} ; \Lambda^{q} \mathbb{C} T^{*}\left(\mathbf{S} N K^{0}\right)\right)$, and

$$
L_{\Theta}=d_{\Theta}-\iota\left(1+\iota \Phi_{x}^{\pi}\right)^{-1}\left(d_{\Theta} \Phi^{\pi}\right) \wedge \partial / \partial x
$$

acting from $\mathcal{C}^{\infty}\left(\mathcal{I} \times \mathcal{T}_{2} ; \Lambda^{q} \mathbb{C} T^{*}\left(\mathbf{S} N K^{0}\right)\right)$ to $\mathcal{C}^{\infty}\left(\mathcal{I} \times \mathcal{T}_{2} ; \Lambda^{q+1} \mathbb{C} T^{*}\left(\mathbf{S} N K^{0}\right)\right)\left[d_{\Theta}\right.$ : exterior derivative on the manifold $\left.\mathbf{S} N K^{0} ; d_{\Theta}=d_{t^{0}}+d_{\theta}\right]$. Using the isomorphism (3.5) we can transform these operators into operators acting on sections of $\mathbf{E}^{(q)}$ over $\mathcal{I} \times\left(\mathcal{T} \backslash K^{0}\right)$ which we continue to call $L_{\rho}$ and $L_{\Theta}$, and decompose the differential operator

$$
L^{\pi}: \mathcal{C}^{\infty}\left(\mathcal{I} \times\left(\mathcal{T} \backslash K^{0}\right) ; \Lambda^{0, q}\right) \rightarrow \mathcal{C}^{\infty}\left(\mathcal{I} \times\left(\mathcal{T} \backslash K^{0}\right) ; \Lambda^{0, q+1}\right)
$$

as a sum $d \rho \wedge L_{\rho}+L_{\Theta}$ : if $f \in \mathcal{C}^{\infty}\left(\mathcal{I} \times\left(\mathcal{T} \backslash K^{0}\right) ; \mathbf{E}^{(q-1)}\right), g \in \mathcal{C}^{\infty}\left(\mathcal{I} \times\left(\mathcal{T} \backslash K^{0}\right) ; \mathbf{E}^{(q)}\right)$, then

$$
\left(d \rho \wedge L_{\rho}+L_{\Theta}\right)(d \rho \wedge f+g)=d \rho \wedge\left(L_{\rho} g-L_{\Theta} f\right)+L_{\Theta} g
$$

Now consider the "first integral" $Z^{\pi}=x+\iota \Phi^{\pi}(x, t)$ of $L^{\pi}: \Phi^{\pi} \equiv 0$ on $K^{0} \Rightarrow$ $\Phi^{\pi}(x, t)=O(|x|+\rho)$ in $\mathcal{I} \times \mathcal{T}$. We may solve the equation $Z^{\pi}=x+\iota \Phi^{\pi}(x, t)$ to get $x=x\left(Z^{\pi}, t\right)\left(\equiv 0\right.$ on $\left.K^{0}\right)$. Later we shall substitute $x=x\left(Z^{\pi}, t\right)$ in analytic functions and in formal power series expansions with respect to $x$, or to $(x, \rho)$. Note, for later reference, that

$$
L_{\rho} Z^{\pi}=0, \quad L_{\Theta} Z^{\pi}=0 .
$$

We shall also use the vector field $L_{0}^{\pi}=\left(1+\iota \Phi_{x}^{\pi}\right)^{-1} \partial / \partial x$ [cf. (1.4) and Section 2].

Lemma 3.2. Let $\{0\} \times K$ be a connected component of $\left(\mathrm{Cl} \Sigma_{1}^{(\kappa)}\right) \cup \cdots \cup\left(\mathrm{Cl} \Sigma_{l_{\kappa}}^{(\kappa)}\right)$ and let $K^{0}$ be the regular part of $K$.

Let $f \in \mathcal{C}^{\infty}\left(\mathcal{I} \times \mathcal{T} ; \Lambda^{0, q}\right)$ have the following properties:

(1) $f \equiv 0$ on $\mathcal{I} \times K^{0}$; 
(2) $f$ vanishes to infinite order on $\{0\} \times\left(K \backslash K^{0}\right)$;

(3) $f$ is a section of $\mathbf{E}^{(q)}$ over $\mathcal{I} \times\left(\mathcal{T} \backslash K^{0}\right)$.

There is $u \in \mathcal{C}^{\infty}\left(\mathcal{I} \times \mathbf{S}^{n} ; \Lambda^{0, q}\right)$ which is a section of $\mathbf{E}^{(q)}$ over $\mathcal{I} \times\left(\mathcal{T} \backslash K^{0}\right)$ and has the following properties:

(A) $\rho L_{\rho} u-f$ vanishes to infinite order on $\{0\} \times K^{0}$;

(B) $u \equiv 0$ on $\mathcal{I} \times K^{0}$;

(C) $\operatorname{supp} u \subset \mathcal{I} \times \mathrm{Cl} \mathcal{T}$

It follows from (A) that $\rho L_{\rho} u$, a priori defined for $\rho \neq 0$, extends as a $\mathcal{C}^{\infty}$ section of $\Lambda^{0, q-1}$ to the whole of $\mathcal{I} \times \mathbf{S}^{n}$, of course supported in $\mathcal{I} \times \mathrm{Cl} \mathcal{T}$ by $(\mathrm{C})$.

Proof. We exploit the property that any $\mathcal{C}^{\infty}$ function in the open subset $\mathcal{T}_{1}$ of $N K^{0}$, or for that matter any $\mathcal{C}^{\infty}$ section of a smooth vector bundle over $\mathcal{T}_{1}$, has a (formal) Taylor expansion at the zero section in powers of the Euclidean distance $\rho$. The Taylor coefficients are functions (resp., sections) defined in the sphere bundle $\mathbf{S} N K^{0}$. In our case, taking into account the presence of the variable $x$ and making the change of indeterminate $x \rightarrow Z^{\pi}(x, t)$, we are allowed to deal with the Taylor expansion of $f$ with respect to $\left(Z^{\pi}, \rho\right)$ about $(0,0), \sum_{a, b=0}^{\infty}\left(Z^{\pi}\right)^{a} \rho^{b+1} f_{a(b+1)}(\Theta)$. Each section $f_{a b}(\Theta)=\left.\frac{1}{a ! b !}\left(L_{0}^{\pi}\right)^{a} L_{\rho}^{b} f\right|_{x=\rho=0}$ is a smooth section of $\Lambda^{q} \mathbb{C} T^{*} \mathbf{S} N K^{0}$ and is flat on $K \backslash K^{0}$. Let us state precisely what the latter means. We use the fact that $\rho^{b} f_{a b}(\Theta)$ extends as a $\mathcal{C}^{\infty}$ section of $\Lambda^{q} \mathbb{C} T^{*} N K^{0}$ over $\mathcal{T}_{1}$ (and not just over $\left.\mathcal{T}_{1} \backslash 0\right)$. To say that $f_{a b}(\Theta)$ is flat on $K \backslash K^{0}$ is the same as saying that $\rho^{b} f_{a b}(\Theta)$ vanishes to infinite order on $K \backslash K^{0}$. More explicitly, we transfer $\rho^{b} f_{a b}(\Theta)$ as a $\mathcal{C}^{\infty}$ $q$-form $\varphi_{a b}(t)$ in $\mathcal{T}$ via the exponential map. Then (cf. Section 2) to each integer $N>0$ and each differential operator $P\left(t, \partial_{t}\right)$ with $\mathcal{C}^{\infty}$ coefficients in $\mathbf{S}^{n}$ there is a constant $C_{N, P}>0$ such that

$$
\left|P\left(t, \partial_{t}\right) \varphi_{a b}(t)\right| \leq C_{N, P}\left[\operatorname{dist}\left(t, K \backslash K^{0}\right)\right]^{N}, \quad \forall t \in \mathcal{T} .
$$

At the left $|\cdot|$ is the Riemannian norm in the exterior algebra and $P\left(t, \partial_{t}\right)$ acts on the coefficients of $\varphi_{a b}$ in local charts of $\mathbf{S}^{n}$. Thanks to (3.6) we have

$$
L_{\rho}\left(\sum_{a+b=0}^{\infty} \frac{1}{b+1}\left(Z^{\pi}\right)^{a} \rho^{b+1} f_{a(b+1)}(\Theta)\right)=\sum_{a+b=0}^{\infty}\left(Z^{\pi}\right)^{a} \rho^{b} f_{a(b+1)}(\Theta) .
$$

We select (cf. proof of Lemma 3.1) a function $\chi \in \mathcal{C}^{\infty}(\mathbb{R}), \chi(\tau)=1$ if $\tau<1, \chi(\tau)=0$ if $\tau>2$, and positive numbers $\epsilon_{\nu} \searrow 0(\nu=0,1,2, \ldots)$ such that

$$
\sum_{a+b=0}^{\infty} \frac{1}{b+1} \chi\left((|x|+\rho) / \epsilon_{a}\right) \chi\left(\rho / \epsilon_{b} \delta\left(t^{0}\right)\right)\left(Z^{\pi}\right)^{a} \rho^{b+1} f_{a(b+1)}(\Theta)
$$

converges to a section $u \in \mathcal{C}^{\infty}\left(\mathcal{I} \times \mathcal{T} ; \Lambda^{0, q}\right)$-thanks to the fact that the products $\rho^{b} f_{a b}(\Theta)$ are smooth sections in $\mathcal{T}$. As a matter of fact $\rho^{b} f_{a b}(\Theta)$ is a smooth section of $\mathbf{E}^{(q)}$ for $\rho>0$ (the region in which the bundle $\mathbf{E}^{(q)}$ is defined), and thus the restriction of $u$ to $\mathcal{I} \times\left(\mathcal{T} \backslash K^{0}\right)$ is a section of $\mathbf{E}^{(q)}$. Note also that if $\epsilon_{0}$ is sufficiently small, then the supports of all the functions $\chi\left(x / \epsilon_{a}\right) \chi\left(\rho / \epsilon_{b} \delta\left(t^{0}\right)\right)$ are contained in $\mathcal{I} \times\left[\mathcal{T} \cup\left(K \backslash K^{0}\right)\right]$.

To show that $u$ vanishes to infinite order on $\mathcal{I} \times\left(K \backslash K^{0}\right)$ we avail ourselves of (3.3) and of the fact that the sections $f_{a b}(\Theta)$ are flat on $K \backslash K^{0}$ : given any $N, k \in \mathbb{Z}_{+}$there is a constant $A_{a b k}^{N}>0$ such that, for all $a, b \in \mathbb{Z}_{+}$and all $j \leq k$,

$$
\left|f_{a(b+1)}\left(t^{0}, \theta\right)\right| / \delta\left(t^{0}\right)^{j} \leq A_{a b k}^{N} \operatorname{dist}\left(t^{0}, K \backslash K^{0}\right)^{N}, \quad \forall\left(t^{0}, \theta\right) \in \mathbf{S} N K^{0} .
$$


First, let $\left(L_{0}^{\pi}\right)^{k}\left(L_{\rho}\right)^{l}$ act on the series (3.8). There are positive constants $C_{a, b, k, l}^{i, j}$, $C_{a, b, k, l}^{\prime}$ such that

$$
\begin{aligned}
& \left|\left(L_{0}^{\pi}\right)^{k}\left(L_{\rho}\right)^{l}\left\{\sum_{a+b=0}^{\infty} \frac{1}{b+1} \chi\left((|x|+\rho) / \epsilon_{a}\right) \chi\left(\rho / \epsilon_{b} \delta\left(t^{0}\right)\right)\left(Z^{\pi}\right)^{a} \rho^{b+1} f_{a(b+1)}(\Theta)\right\}\right| \\
& \quad \leq \sum_{\substack{0 \leq i \leq k \\
k \leq a+i}} \sum_{\substack{0 \leq j \leq l \\
l \leq b+j}} C_{a, b, k, l}^{i, j} \epsilon_{a}^{-i} \epsilon_{b}^{-j}\left|\chi^{(i)}\left((|x|+\rho) / \epsilon_{a}\right)\right| \cdot\left|\chi^{(j)}\left(\rho / \epsilon_{b} \delta\left(t^{0}\right)\right)\right| \\
& \quad \leq\left\{\sum_{a+b=0}^{\infty} C_{a, b, k, l}^{\prime} A_{a b k}^{N} \epsilon_{a}^{a-k} \epsilon_{b}^{b+1-l}\right\} \operatorname{dist}\left(t^{0}, K \backslash K^{0}\right)^{N} .
\end{aligned}
$$

We require

$$
C_{a, b, k, l}^{\prime} A_{a b k}^{N} \epsilon_{a}^{a-k} \epsilon_{b}^{b-l} \leq 2^{-a-b} \quad \text { if } k+l \leq N<a+b,
$$

which yields, for some constant $B_{N}>0$,

$$
\begin{aligned}
& \left|\left(L_{0}^{\pi}\right)^{k}\left(L_{\rho}\right)^{l}\left\{\sum_{a+b=0}^{\infty} \frac{1}{b+1} \chi\left((|x|+\rho) / \epsilon_{a}\right) \chi\left(\rho / \epsilon_{b} \delta\left(t^{0}\right)\right)\left(Z^{\pi}\right)^{a} \rho^{b+1} f_{a(b+1)}(\Theta)\right\}\right| \\
& \leq B_{N} \operatorname{dist}\left(t^{0}, K \backslash K^{0}\right)^{N}
\end{aligned}
$$

in $\mathcal{T}$. Now let $P$ be an arbitrary differential operator with $\mathcal{C}^{\infty}$ coefficients on $\mathbf{S}^{n}$ and let $\left(L_{0}^{\pi}\right)^{k} P(t, \partial / \partial t)$ act on each term of the series (3.8). We repeat the preceding argument. We can compensate for the negative powers of $\delta$ Thanks To Hypothesis (3.3) and to (3.7) applied to the coefficients $f_{a b}$. We must now impose a condition of the kind (3.9) with constants that depend also on $P$. That the section $u$ fulfills the requirements in Lemma 3.2 is easily checked.

The forthcoming argument will be predicated on the property that the pullback of every form $\left(L_{0}^{\pi}\right)^{k} f\left(k \in \mathbb{Z}_{+}\right)$to each stratum of $\mathcal{F}^{\pi}(0)$ is exact. The following lemma will be applied:

Lemma 3.3. Let $K$ be as in Lemma 3.2. Let a form $f \in \mathcal{C}^{\infty}\left(\mathcal{I} \times \mathbf{S}^{n} ; \Lambda^{0, p}\right)$ have the following properties:

$$
\begin{gathered}
f \text { vanishes to infinite order on }\{0\} \times\left(K \backslash K^{0}\right) ; \\
L^{\pi} f \text { vanishes to infinite order on }\{0\} \times K ; \\
\forall k \in \mathbb{Z}_{+} \text {, the pullback of }\left(L_{0}^{\pi}\right)^{k} f \text { to }\{0\} \times K^{0} \text { is exact. }
\end{gathered}
$$

Then there is $v \in \mathcal{C}^{\infty}\left(\mathcal{I} \times \mathbf{S}^{n} ; \Lambda^{0, p-1}\right)$ such that $\tilde{f}=f-L^{\pi} v$ has Properties (3.10) and (3.11), as well as the following one:

$$
\forall k \in \mathbb{Z}_{+} \text {, the pullback of }\left(L_{0}^{\pi}\right)^{k} \tilde{f} \text { to }\{0\} \times K^{0} \text { vanishes identically. }
$$

Proof. Call $\mathfrak{p}$ the map $\mathcal{T} \supset \sigma_{t^{0}} \ni t \rightarrow t^{0} \in K^{0}$ and $\varphi^{*}$ the pullback to $\{0\} \times$ $K^{0} ; \varphi^{*} \mathfrak{p}^{*}=$ Identity. Hypothesis (3.12) implies that

$$
f_{k}=\mathfrak{p}^{*} \varphi^{*}\left(L_{0}^{\pi}\right)^{k} f \in \mathcal{C}^{\infty}\left(\mathcal{T} ; \Lambda^{p} \mathbb{C} T^{*} \mathbf{S}^{n}\right)
$$

is exact, and (3.10) that $f_{k}$ is flat at $K \backslash K^{0}$. By (3.3) there are countable triangulations of a neighborhood of $(\mathrm{Cl} \mathcal{T}) \backslash\left(K \backslash K^{0}\right)$ with a good control of the size of the 
simplices in terms of their distance to $K \backslash K^{0}$. We use the method of [18] to integrate the equation $d_{t} \omega_{k}=f_{k}$ with estimates ensuring that $\omega_{k} \in \mathcal{C}^{\infty}\left(\mathrm{Cl} \mathcal{T} ; \Lambda^{p} \mathbb{C} T^{*} \mathbf{S}^{n}\right)$ and that $\omega_{k}$ is flat at $K \backslash K^{0}$. We can now extend $\omega_{k}$ as a $\mathcal{C}^{\infty}(p-1)$-form to the whole sphere $\mathbf{S}^{n-1}$. To do this we introduce a finite covering of $\mathrm{Cl} \mathcal{T}$ by open sets in which the boundary $\partial \mathcal{T}$ can be desingularized and $\Lambda^{p-1} T^{*} \mathbf{S}^{n}$ admits smooth bases. We extend across $\partial \mathcal{T}$ each coefficient of $\omega_{k}$ in one of these bases. Away from $K \backslash K^{0}$ our integration of the equation $d_{t} \omega_{k}=f_{k}$ is carried out in simplices that cross the boundary $\partial \mathcal{T}$ and thus yields an automatic extension. At points of $K \backslash K^{0}$ the coefficients are flat and desingularization transforms the geometry into that of corners.

We identify the extension of $\omega_{k}$ to a differential form $\varpi_{k}$ on $\mathcal{I} \times \mathbf{S}^{n}$ via the pullback $(x, t) \rightarrow t$ and we define

$$
v=\sum_{k=0}^{\infty} \frac{1}{k !} \chi\left((|x|+\rho) / \epsilon_{k}\right)\left(Z^{\pi}\right)^{k} \varpi_{k} \in \mathcal{C}^{\infty}\left(\mathcal{I} \times \mathbf{S}^{n} ; \Lambda^{0, p-1}\right),
$$

where the variable $\rho$ and the cutoff function $\chi$ have the same meaning as in the proof of Lemma 3.2, and the selection of the numbers $\epsilon_{k}\left(>\epsilon_{k+1} \rightarrow+0\right)$ is similar to their selection in that proof. Observe that

$$
L^{\pi} v \cong \sum_{k=0}^{\infty} \frac{1}{k !} \chi\left((|x|+\rho) / \epsilon_{k}\right)\left(Z^{\pi}\right)^{k} f_{k}
$$

modulo forms that vanish to infinite order on $\{0\} \times K^{0} ; \operatorname{supp} L^{\pi} v \subset \mathcal{I} \times\left[\mathcal{T} \cup\left(K \backslash K^{0}\right)\right]$ if the numbers $\epsilon_{k}$ are sufficiently small; and, as a consequence, the coefficients of $f-L^{\pi} v$ vanish to infinite order on $\{0\} \times\left(K \backslash K^{0}\right)$.

Property (3.11) is unchanged if we substitute $f-L^{\pi} v$ for $f$. Finally, for any $k=0,1, \ldots, \varphi^{*}\left(L_{0}^{\pi}\right)^{k} L^{\pi} v=\varphi^{*}\left(L_{0}^{\pi}\right)^{k} f$ since $Z^{\pi} \equiv 0$ on $K^{0}$.

Lemma 3.3 reduces the situation to the one dealt with in the following

Lemma 3.4. To each $f \in \mathcal{C}^{\infty}\left(\mathcal{I} \times \mathbf{S}^{n} ; \Lambda^{0, p}\right)$ with Properties (3.10), (3.11) and $\left(3.12^{\prime}\right)$ there is $u \in \mathcal{C}^{\infty}\left(\mathcal{I} \times \mathbf{S}^{n} ; \Lambda^{0, p-1}\right)$ such that $L^{\pi} u-f$ vanishes to infinite order on $\{0\} \times K$.

Proof. We use the same notation as in the proof of Lemma 3.2. The direct sum decomposition (3.4) allows us to write $f=f_{0}+d \rho \wedge \rho^{-1} f_{1}$ in $\mathcal{I} \times\left(\mathcal{T} \backslash K^{0}\right)$ with $f_{0} \in \mathcal{C}^{\infty}\left(\mathcal{I} \times\left(\mathcal{T} \backslash K^{0}\right) ; \mathbf{E}^{(p)}\right)$ and $f_{1} \in \mathcal{C}^{\infty}\left(\mathcal{I} \times\left(\mathcal{T} \backslash K^{0}\right) ; \mathbf{E}^{(p-1)}\right)$. The following consequence of the smoothness of $f$ is crucial for the rest of the argument: $f_{1} \equiv 0$ when $\rho=0$ and $f_{1}$ extends as a smooth $(p-1)$-form to $\mathcal{I} \times \mathcal{T}$. To prove this claim one can, by (3.5), identify $f_{1}$ to a section of $\Lambda^{p-1} \mathbb{C} T^{*} \mathbf{S} N K^{0}$ which depends smoothly on $\rho>0$, and then use local coordinates in the (spherical) fibres of $\mathbf{S} N K^{0}$.

Furthermore, (3.10) implies that both $f_{0}$ and $f_{1}$ are flat on $\{0\} \times\left(K \backslash K^{0}\right)$. Here we are extending slightly the concept of flatness introduced in the proof of Lemma 3.2: now it also involves the variable $x$ and (3.7) must be replaced by estimates of the kind

$$
\left|\partial_{x}^{k} P\left(t, \partial_{t}\right) \tilde{f}(x, t)\right| \leq C_{N, k, P}\left[|x|+\operatorname{dist}\left(t, K \backslash K^{0}\right)\right]^{N}, \quad \forall(x, t) \in \mathcal{I} \times \mathcal{T},
$$

for $k=0,1,2, \ldots$

We apply Lemma 3.2 to $f_{1}$ to get a form $u \in \mathcal{C}^{\infty}\left(\mathcal{I} \times \mathbf{S}^{n} ; \Lambda^{0, p-1}\right)$ with the following properties: $\operatorname{supp} u \subset \mathcal{I} \times(\mathrm{Cl} \mathcal{T}) ; u \equiv 0$ on $\mathcal{I} \times K ; L_{\rho} u-\rho^{-1} f_{1}$ vanishes to 
infinite order on $\{0\} \times K$; and by construction the restriction of $u$ to $\mathcal{I} \times\left(\mathcal{T} \backslash K^{0}\right)$ is a section of $\mathbf{E}^{(p-1)}$.

For $p=n$ the argument stops here: $f_{0} \equiv 0, L^{\pi} u=d \rho \wedge L_{\rho} u$ and thus $L^{\pi} u-f$ vanishes to infinite order on $\{0\} \times K$. In the remainder of the proof we assume $p \leq n-1$.

Observe that

$$
\left(f-L^{\pi} u\right)-\left(f_{0}-L_{\Theta} u\right)=d \rho \wedge\left(\rho^{-1} f_{1}-L_{\rho} u\right)
$$

vanishes to infinite order on $\{0\} \times K$. One can view $f_{0}-L_{\Theta} u$ as a $\mathcal{C}^{\infty}$ section of $\mathbf{E}^{(p)}$ and even as a $\mathcal{C}^{\infty}$ section of $\Lambda^{p} \mathbb{C} T^{*}\left(N K^{0}\right)$ over $\mathcal{I} \times\left(\mathcal{T} \backslash K^{0}\right)$-by (3.5). With this interpretation the operators $L_{0}^{\pi}$ and $L_{\rho}$ act on $f_{0}-L_{\Theta} u$. For each $(a, b) \in \mathbb{Z}_{+}^{2}$, define

$$
F_{a b}=\frac{1}{a ! b !} \lim _{(x, \rho) \rightarrow(0,0)}\left(L_{0}^{\pi}\right)^{a} L_{\rho}^{b}\left(f-L^{\pi} u\right) .
$$

The forms $F_{a b}$ depend only on $\Theta=\left(t^{0}, \theta\right) \in \mathbf{S} N K^{0}$, and only on $t^{0}$ when $b=0$. We have

$$
\lim _{(x, \rho) \rightarrow(0,0)}\left(L_{0}^{\pi}\right)^{a} L_{\rho}^{b}\left(f-L^{\pi} u\right)=\lim _{(x, \rho) \rightarrow(0,0)}\left(L_{0}^{\pi}\right)^{a} L_{\rho}^{b}\left(f_{0}-L_{\Theta} u\right) .
$$

Strictly speaking, the formal series

$$
F^{\#}=\sum_{a+b=0}^{\infty}\left(Z^{\pi}\right)^{a} \rho^{b} F_{a b}
$$

is equal to the Taylor expansion of $f-L^{\pi} u$ with respect to $\left(Z^{\pi}, \rho\right)$ about $(0,0)$.

It follows from hypothesis $\left(3.12^{\prime}\right)$ (with $f$ substituted for $\tilde{f}$ ) that $\left(L_{0}^{\pi}\right)^{a} f_{0}$ tends to zero as $\rho+|x| \rightarrow 0$. On the other hand, $\left.\left.u\right|_{\rho=0} \equiv 0 \Rightarrow \lim _{\rho \rightarrow 0}\left(L_{0}^{\pi}\right)^{a} L_{\Theta} u\right|_{\rho=0} \equiv 0$. We conclude that $F_{a 0} \equiv 0$ for all $a \in \mathbb{Z}_{+}$.

We also make use of the Taylor expansion of the coefficients of the vector field $L_{\rho}$, which provides us with the formal vector field

$$
L_{\rho}^{\#}=\partial / \partial \rho+\sum_{a+b=0}^{\infty}\left(Z^{\pi}\right)^{a} \rho^{b} c_{a b} L_{0}^{\pi} .
$$

The coefficients $c_{a b}$ depend solely on $\Theta \in \mathbf{S} N K^{0}$. By (3.11) $L^{\pi} f=L^{\pi}\left(f-L^{\pi} u\right)$ vanishes to infinite order when $x=\rho=0$. We derive that $L_{\rho}^{\#} F^{\#}=0$, i.e.,

$L_{\rho}^{\#} \sum_{a+b=0}^{\infty}\left(Z^{\pi}\right)^{a} \rho^{b} F_{a b}=\sum_{a+b=0}^{\infty}\left(b\left(Z^{\pi}\right)^{a} \rho^{b-1} F_{a b}+\sum_{i+j=0}^{\infty} a\left(Z^{\pi}\right)^{a+i-1} \rho^{b+j} c_{i j} F_{a b}\right)=0$ or, equivalently,

$$
(b+1) F_{a(b+1)}=-\sum_{i=0}^{a} \sum_{j=0}^{b}(a-i+1) c_{i j} F_{(a-i+1)(b-j)} .
$$

Since $F_{k 0} \equiv 0$ for each $k \in \mathbb{Z}_{+}$, necessarily $F_{a b} \equiv 0$ for all $a, b \in \mathbb{Z}_{+}$. This proves that $f-L^{\pi} u$ vanishes to infinite order at $x=\rho=0$.

We are now in a position to prove the following part of Lemma 2.1:

Lemma 3.5. If $f \in \mathcal{C}^{\infty}\left(\mathcal{I} \times \mathbf{S}^{n} ; \Lambda^{0, p}\right)$ is $L^{\pi}$-closed and if, for any $k=0,1, \ldots$, the pullback of $\left(L_{0}^{\pi}\right)^{k} f$ to any smooth submanifold of $\mathcal{F}^{\pi}(0)$ is exact, then there is $u \in \mathcal{C}^{\infty}\left(\mathcal{I} \times \mathbf{S}^{n} ; \Lambda^{0, p-1}\right)$ such that $f-L^{\pi} u$ vanishes to infinite order on $\mathcal{F}^{\pi}(0)$. 
Proof. By Lemma 3.1 the claim is evidently true when $\mathcal{F}^{\pi}(0)=\{0\} \times \mathbf{S}^{n}$. When $\mathcal{F}^{\pi}(0) \neq\{0\} \times \mathbf{S}^{n}$ let $\Sigma_{1}, \ldots, \Sigma_{s_{0}}$ be the compact strata of $\mathcal{F}^{\pi}(0)$ [for the natural stratification of $\left.\mathcal{F}^{\pi}(0)\right]$. For each $i=1, \ldots, s_{0}$, the conditions (3.10), (3.11), (3.12) are satisfied with $K=\Sigma_{i}$. By combining Lemmas 3.3 and 3.4 we find $v_{i} \in$ $\mathcal{C}^{\infty}\left(\mathcal{I} \times \mathbf{S}^{n} ; \Lambda^{0, p-1}\right)$ such that $f-L v_{i}$ vanishes to infinite order on $\Sigma_{i}$. Let $\chi_{i} \in$ $\mathcal{C}^{\infty}\left(\mathcal{I} \times \mathbf{S}^{n}\right), \chi_{i} \equiv 1$ in a neighborhood of $\Sigma_{i}, \chi_{i} \equiv 0$ in a neighborhood of every $\Sigma_{j}, 1 \leq j \leq s_{0}, j \neq i$. It is clear that $f-L\left(\sum_{i=1}^{s_{0}} \chi_{i} v_{i}\right)$ vanishes to infinite order on $\bigcup_{i=1}^{s_{0}} \Sigma_{i}$.

Now suppose $f$ vanishes to infinite order on any stratum of $\mathcal{F}^{\pi}(0)$ whose codimension is $>\kappa \geq 1$. By the preceding argument we know this to be the case if $\kappa$ is equal to the maximum of the codimension of the noncompact strata (in which case $\kappa<n)$. Now let $K_{1}, \ldots, K_{s_{\kappa}}$ be the connected components of the set $\bar{\Sigma}_{1}^{(\kappa)} \cup \cdots \cup \bar{\Sigma}_{l_{\kappa}}^{(\kappa)}$. [We are changing the notation a bit: above, these connected components would have been called $\{0\} \times K_{i}$ but this precision is not needed here.] For each $i=1, \ldots, s_{\kappa}$ we can find $v_{i} \in \mathcal{C}^{\infty}\left(\mathcal{I} \times \mathbf{S}^{n} ; \Lambda^{0, p-1}\right)$ such that $f-L v_{i}$ is flat on $K_{i}$. Let $\chi_{i} \in \mathcal{C}^{\infty}\left(\mathcal{I} \times \mathbf{S}^{n}\right), \chi_{i} \equiv 1$ in a neighborhood of $K_{i}, \chi_{i} \equiv 0$ in one of each $K_{j}, 1 \leq j \leq s_{\kappa}, j \neq i$. It is clear that $f-L\left(\sum_{i=1}^{s_{\kappa}} \chi_{i} v_{i}\right)$ is flat on $\bigcup_{i=1}^{s_{\kappa}} K_{i}$. Thus descending induction on $\kappa$ proves the assertion.

Henceforth we assume that the form $f$ vanishes to infinite order on the fibre $\mathcal{F}^{\pi}(0)$. We reason in the region $x>0$; the same reasoning applies to the region $x<0$. Call $\Sigma_{i}^{+(\kappa)}\left(i=1, \ldots, q_{\kappa}\right)$ the strata of $\mathcal{A}^{+}$whose codimension is equal to $\kappa$. Below $K$ will be an arbitrary connected component of the union $\left(\mathrm{Cl}_{1}^{+(\kappa)}\right) \cup \cdots \cup$ $\left(\mathrm{Cl} \Sigma_{q_{\kappa}}^{(+\kappa)}\right)$ [all closures are relative to $(-a, a) \times \mathbf{S}^{n}$ ]. Then $K=\left(\mathrm{Cl} \Sigma_{i_{1}}^{+(\kappa)}\right) \cup \cdots \cup$ $\left(\mathrm{Cl} \Sigma_{i_{\alpha}}^{+(\kappa)}\right)\left(1 \leq \alpha \leq q_{\kappa}\right)$; the regular part of $K$ is the $\mathcal{C}^{\omega}$ submanifold of $(0, a) \times \mathbf{S}^{n}$, $K^{0}=\Sigma_{i_{1}}^{+(\kappa)} \cup \cdots \cup \Sigma_{i_{\alpha}}^{+(\kappa)}$; we write $K_{x}^{0}=\left\{t \in \mathbf{S}^{n} ;(x, t) \in K^{0}\right\}(0<x<a)$.

We shall duplicate the argument dealing with $\mathcal{F}^{\pi}(0)$ except that we shall "inject" the coordinate $x$. Thus we introduce a tubular neighborhood $\mathcal{T}$ of $K^{0}$ in $(0, a) \times \mathbf{S}^{n}$ which tapers off at the boundary $K \backslash K^{0}$. For each $\left(x, t^{0}\right) \in K^{0}$ let $\sigma_{x, t^{0}}$ denote the open geodesic $\kappa$-ball in $\mathbf{S}^{n}$ of radius $\delta\left(x, t^{0}\right)$, centered at $t^{0}$, whose tangent space at $t^{0}$ is equal to the normal space to $K_{x}^{0}$. We define

$$
\mathcal{T}=\left\{(x, t) \in(0, a) \times \mathbf{S}^{n} ; \exists t^{0} \in K_{x}^{0} \text { such that } t \in \sigma_{x, t^{0}}\right\} .
$$

We require $\delta\left(x, t^{0}\right)$ to be a positive $\mathcal{C}^{\infty}$ function in $\mathcal{T}$, sufficiently small to ensure that the exponential map in $\mathbf{S}^{n}$ induces a diffeomorphism onto $\mathcal{T}$ of the set

$$
\mathcal{T}_{1}=\left\{\left(x,\left(t^{0}, \nu\right)\right) \in(0, a) \times T \mathbf{S}^{n} ;\left(t^{0}, \nu\right) \in N K_{x}^{0},|\nu|<\delta\left(x, t^{0}\right)\right\} .
$$

On the other hand we don't allow $\delta\left(x, t^{0}\right)$ to be too small: we require

$$
\exists c, \gamma>0 \text { such that } \delta\left(x, t^{0}\right) \geq c\left[\operatorname{dist}\left(\left(x, t^{0}\right), K \backslash K^{0}\right)\right]^{\gamma}, \quad \forall\left(x, t^{0}\right) \in K^{0} .
$$

Such a choice of $\delta\left(x, t^{0}\right)$ is permitted thanks to the properties of analytic sets.

The geodesic distance in $\mathbf{S}^{n}$ between $t^{0} \in K_{x}^{0}$ and $t \in \sigma_{x, t^{0}}, \rho(t)=\operatorname{dist}\left(t, t^{0}\right)$, is the "radial coordinate". Now $\theta$ is the fibre variable and $\Theta=\left(t^{0}, \theta\right)$ the total variable in the normal sphere bundle $\mathbf{S} N K_{x}^{0}$ of $K_{x}^{0}$ in $\mathbf{S}^{n}$. As $x$ ranges over $\mathcal{I}$ these bundles $\mathbf{S} N K_{x}^{0}$ make up a fibre subbundle $\mathbf{S} N^{\prime} K^{0}$ of the normal sphere bundle $\mathbf{S} N K^{0}$ of $K^{0}$ in $\mathcal{I} \times \mathbf{S}^{n}:\left(\left(x, t^{0}\right), \theta\right) \in \mathbf{S} N^{\prime} K^{0} \Leftrightarrow$ the pushdown of $\theta \in \mathbf{S} N_{\left(x, t^{0}\right)} K^{0}$ under the coordinate map $\left(x, t^{0}\right) \rightarrow x$ is equal to zero. We introduce the manifold

$$
\mathcal{T}_{2}=\left\{\left(x,\left(t^{0}, \theta\right), \rho\right) \in \mathbf{S} N^{\prime} K^{0} \times \mathbb{R}_{+} ;\left(t^{0}, \theta\right) \in \mathbf{S} N K_{x}^{0}, 0<\rho<\delta\left(x, t^{0}\right)\right\}
$$


and the natural diffeomorphism of $\mathcal{T}_{2}$ onto $\mathcal{T} \backslash K^{0}$ obtained by composing

$$
\mathcal{T}_{2} \ni\left(x,\left(t^{0}, \theta\right), \rho\right) \rightarrow\left(x, t^{0}, \rho \theta\right) \in \mathcal{T}_{1} \backslash 0 \stackrel{\exp }{\rightarrow} \mathcal{T} \backslash K^{0}
$$

Here the exponential map is defined on the slices corresponding to a fixed $x$.

As before, we call $\mathbf{E}^{(q)}$ the vector subbundle of $\Lambda^{0, q}$ over $\mathcal{T} \backslash K^{0}$ orthogonal (for the Hilbert space structure induced on the fibres by the Riemannian structure of $\left.\mathbf{S}^{n}\right)$ to the vector subbundle $d \rho \wedge \Lambda^{0, q-1}(q \geq 1)$. Agreeing that $\mathbf{E}^{(0)}=\left(\mathcal{T} \backslash K^{0}\right) \times \mathbb{C}$ we have the orthogonal decomposition

$$
\left.\Lambda^{0, q}\right|_{\mathcal{T} \backslash K^{0}}=\mathbf{E}^{(q)} \oplus\left(d \rho \wedge \mathbf{E}^{(q-1)}\right) .
$$

To this decomposition there corresponds the decomposition of the differential operator $L^{\pi}=d \rho \wedge L_{\rho}+L_{\Theta}$ used earlier, with $L_{\rho}$ and $L_{\Theta}$ acting on sections, over $\mathcal{T} \backslash K^{0}$, of $\mathbf{E}^{(q-1)}$ and $\mathbf{E}^{(q)}$ respectively.

Let $W$ denote the pullback of $Z$ under the blow-up map $\mathcal{T}_{2} \rightarrow \mathcal{T} \backslash K^{0}$; define the vector bundle over $\mathcal{T}_{2}, \Lambda^{\prime 0, q}=\Lambda^{q} \mathbb{C} T^{*} \mathcal{T}_{2} /\left(d W \wedge \Lambda^{q-1} \mathbb{C} T^{*} \mathcal{T}_{2}\right)$. The blow-up map $\mathcal{T}_{2} \rightarrow \mathcal{T} \backslash K^{0}$ defines an isomorphism of $\Lambda^{0, q}$ onto $\Lambda^{\prime 0, q}$. The exterior derivative in $\mathbf{S} N^{\prime} K^{0} \times \mathbb{R}_{+}$induces a differential operator $L^{\prime}: \mathcal{C}^{\infty}\left(\mathcal{T}_{2} ; \Lambda^{\prime 0, q}\right) \rightarrow \mathcal{C}^{\infty}\left(\mathcal{T}_{2} ; \Lambda^{\prime 0, q+1}\right)$. The factorization $\mathbf{S} N^{\prime} K^{0} \times \mathbb{R}$ leads to a decomposition of the exterior algebra $\Lambda \mathbb{C} T^{*}\left(\mathbf{S} N^{\prime} K^{0} \times \mathbb{R}\right)$ and, by way of consequence, of $\Lambda^{\prime 0, q}(q=0,1, \ldots)$. The Riemannian metric of $\mathbf{S}^{n}$ or, if one prefers, of $\mathcal{I} \times \mathbf{S}^{n}$, induces a Riemannian metric on the manifold $\mathbf{S} N^{\prime} K^{0}$ and thereby a structure of Hilbert vector bundles on the exterior powers $\Lambda^{q} \mathbb{C} T^{*}\left(\mathbf{S} N^{\prime} K^{0} \times \mathbb{R}\right)$ and on their quotients $\Lambda^{\prime 0, q}$. Let $\mathbf{E}^{\prime(q)}$ denote the orthogonal of $d \rho \wedge \Lambda^{\prime 0, q-1}$ in $\Lambda^{\prime 0, q}$; it is checked at once that the isomorphism $\Lambda^{0, q} \cong \Lambda^{\prime 0, q}$ induces an isomorphism $\mathbf{E}^{\prime(q)} \cong \mathbf{E}^{(q)}$. Of course, $\Lambda^{\prime 0, q}=\mathbf{E}^{\prime(q)} \oplus\left(d \rho \wedge \mathbf{E}^{\prime(q-1)}\right)$. This decomposition yields a decomposition $L^{\prime}=L_{\Theta}^{\prime}+d \rho \wedge L_{\rho}^{\prime} ; L^{\pi}, L_{\Theta}$ and $L_{\rho}$ are the transforms of $L^{\prime}, L_{\Theta}^{\prime}$ and $L_{\rho}^{\prime}$ respectively, under the isomorphism $\Lambda^{0, q} \cong \Lambda^{\prime 0, q}$.

Below the operator $L_{0}^{\pi}$ will act on sections of $\mathbf{E}^{(q)}$. This action must be defined precisely as follows: let $f$ be a $\mathcal{C}^{\infty}$ section of $\Lambda^{0, q}$ over some open subset $\mathcal{U}$ of $\mathcal{I} \times \mathbf{S}^{n}$, and let $f_{0} \in \mathcal{C}^{\infty}\left(\mathcal{U} ; \Lambda^{q} \mathbb{C} T^{*}\left(\mathcal{I} \times \mathbf{S}^{n}\right)\right)$ represent $f$. If $f_{1}$ is any $\mathcal{C}^{\infty}$ section of $\Lambda^{q+1} \mathbb{C} T^{*}\left(\mathcal{I} \times \mathbf{S}^{n}\right)$ over $\mathcal{U}$ representing $L^{\pi} f$, then $d f_{0}-f_{1}=d Z^{\pi} \wedge f_{2}$ for some $f_{2} \in \mathcal{C}^{\infty}\left(\mathcal{U} ; \Lambda^{q} \mathbb{C} T^{*}\left(\mathcal{I} \times \mathbf{S}^{n}\right)\right)$. The equivalence class of $f_{2}$ in $\Lambda^{0, q}$ is equal to $L_{0}^{\pi} f$. Since $L_{\rho} Z^{\pi} \equiv L_{0}^{\pi} \rho \equiv 0$ and of course $L_{0}^{\pi} Z^{\pi} \equiv L_{\rho} \rho \equiv 1$, we conclude that $L_{0}^{\pi}$ maps $\mathcal{C}^{\infty}\left(\mathcal{T} \backslash K^{0} ; \mathbf{E}^{(q)}\right)$ into itself.

All this can be translated in $\mathcal{T}_{2}$ for sections of $\mathbf{E}^{\prime(q)}$ yielding a differential operator $L_{0}^{\prime \pi}: \mathcal{C}^{\infty}\left(\mathcal{T}_{2} ; \mathbf{E}^{\prime(q)}\right) \rightarrow \mathcal{C}^{\infty}\left(\mathcal{T}_{2} ; \mathbf{E}^{\prime(q)}\right) ; L_{0}^{\prime \pi}$ can be regarded as an operator depending smoothly on the parameter $\rho$ (in a neighborhood of zero whose size depends on the base point in $\mathcal{T}_{2}$ ) and, as such, admits a Taylor expansion about $\rho=0$. We can retransform $L_{0}^{\prime \pi}$ into $L_{0}^{\pi}$ and thus obtain the Taylor expansion of the latter with respect to $\rho$ about zero.

These remarks will now be used in the proof of the analogue of Lemma 3.2:

Lemma 3.6. Let $K$ be a connected component of $\left(\mathrm{Cl}_{1}^{(\kappa)}\right) \cup \cdots \cup\left(\mathrm{Cl} \Sigma_{l_{\kappa}}^{(\kappa)}\right)$ with regular part $K^{0}$. Let $f \in \mathcal{C}^{\infty}\left(\mathcal{I} \times \mathcal{T} ; \Lambda^{0, q}\right)$ have the following properties:

(1) $f \equiv 0$ on $K$;

(2) $f$ vanishes to infinite order on $K \backslash K^{0}$;

(3) $f$ is a section of $\mathbf{E}^{(q)}$ over $\mathcal{T} \backslash K^{0}$.

Then there is $u \in \mathcal{C}^{\infty}\left(\mathcal{I} \times \mathbf{S}^{n} ; \Lambda^{0, q}\right)$ which is a section of $\mathbf{E}^{(q)}$ over $\mathcal{T} \backslash K^{0}$ and has the following properties: 
(A) $\rho L_{\rho} u-f$ vanishes to infinite order on $K^{0}$;

(B) $u \equiv 0$ on $K$;

(C) $u$ is a section of $\mathbf{E}^{(q)}$ over $\mathcal{T} \backslash K^{0}$;

(D) $\operatorname{supp} u \subset \mathrm{Cl} \mathcal{T}$.

Proof. Pullback to $\mathcal{T}_{2}$ shows (cf. beginning of the proof of Lemma 3.2) that we have the right to form the Taylor expansion $\sum_{k=1}^{\infty} \rho^{k} f_{k}$ of $f$ with respect to $\rho$ about zero. For each $k=1,2, \ldots, \rho^{k} f_{k}(x, \Theta)=\frac{1}{k !} \rho^{k} \lim _{\rho \rightarrow 0} \partial^{k} f / \partial \rho^{k}$ is a smooth section of $\Lambda^{0, q-1}$ over $\mathcal{T}$ and vanishes to infinite order on $K \backslash K^{0}$; it is a section of $\mathbf{E}^{(q)}$ over $\mathcal{T} \backslash K^{0}$. Let $\sum_{k=0}^{\infty} \rho^{k} c_{k}(x, \Theta) \partial / \partial x$ denote the Taylor expansion of the operator $\iota\left(\partial \Phi^{\pi} / \partial \rho\right) L_{0}^{\pi}$ (see above). It is important to note that, for each $k$, $c_{k}(x, \Theta) \partial / \partial x$ defines an endomorphism of $\mathcal{C}^{\infty}\left(\mathcal{T} \backslash K^{0} ; \mathbf{E}^{(q)}\right)$; and also that, except in trivial cases, $\partial \Phi^{\pi} / \partial \rho$ does not extend as a $\mathcal{C}^{\infty}$ function to $\mathcal{T}$, but that $\rho \partial \Phi^{\pi} / \partial \rho$ does. It follows that each $\rho^{k+1} c_{k}(x, \Theta)$ extends as a $\mathcal{C}^{\infty}$ function in $\mathcal{T}$. This said we seek a power series $\sum_{k=1}^{\infty} \rho^{k} u_{k}(x, \Theta)$ such that $\rho^{k} u_{k}(x, \Theta) \in \mathcal{C}^{\infty}\left(\mathcal{T} ; \Lambda^{0, q}\right)$ for all $k$, and

$$
\left(\partial / \partial \rho-\sum_{i=0}^{\infty} \rho^{i} c_{i} \partial / \partial x\right)\left(\sum_{j=1}^{\infty} \rho^{j} u_{j}\right)=\sum_{k=1}^{\infty} \rho^{k-1} f_{k} .
$$

We use recurrence on $k=1,2, \ldots$ to solve the equations

$$
k u_{k}=\sum_{a+b=k-1} c_{a} \partial u_{b} / \partial x+f_{k}
$$

This implies $u_{k} \in \mathcal{C}^{\infty}\left(\mathcal{T} \backslash K^{0} ; \mathbf{E}^{(q)}\right)$ and

$$
k \rho^{k} u_{k}=\sum_{a+b=k-1}\left(\rho^{a+1} c_{a}\right) \partial\left(\rho^{b} u_{b}\right) / \partial x+\rho^{k} f_{k} .
$$

Induction on $k$ and the smoothness of $\rho^{a+1} c_{a}$ and $\rho^{k} f_{k}$ implies that $\rho^{k} u_{k}$ defines a $\mathcal{C}^{\infty}$ section of $\Lambda^{0, q}$ in $\mathcal{T}$ for every $k=1,2, \ldots$ We introduce the usual cutoff function $\chi \in \mathcal{C}^{\infty}(\mathbb{R}), \chi(\tau)=1$ if $\tau<1, \chi(\tau)=0$ if $\tau>2$, and select positive numbers $\epsilon_{\nu} \searrow 0(\nu=0,1,2, \ldots)$ such that $\sum_{k=1}^{\infty} \chi\left(\rho / \epsilon_{k} \delta\left(x, t^{0}\right)\right) \rho^{k} u_{k}\left(x, t^{0}, \theta\right)$ converges to a $\mathcal{C}^{\infty}$ section $u$ of $\Lambda^{0, q}$ in $\mathcal{T}$ which vanishes to infinite order on $K \backslash K^{0}$. We take $\epsilon_{0}$ sufficiently small to ensure that the supports of all the functions $\chi\left(\rho / \epsilon_{k} \delta\left(x, t^{0}\right)\right)$ are contained in $\mathcal{T} \cup\left(K \backslash K^{0}\right)$. The claim follows in the usual manner from (3.14) and from the flatness of the $f_{k}(x, \Theta)$ on $K \backslash K^{0}$. Furthermore we can extend $u$ to $\mathcal{I} \times \mathbf{S}^{n}$ setting $u \equiv 0$ in the complement of $\mathcal{T}$ (in particular in the region $x \leq 0$ ).

Lemma 3.7. Let $K$ be as in Lemma 3.6. Suppose that $f \in \mathcal{C}^{\infty}\left(\mathcal{I} \times \mathbf{S}^{n} ; \Lambda^{0, p}\right)$ is $L^{\pi}$-closed, that its pullback to any $\mathcal{C}^{\infty}$ submanifold of any fibre of $Z^{\pi}$ is exact and that $f$ vanishes to infinite order on $K \backslash K^{0}$. Then there is $u_{K} \in \mathcal{C}^{\infty}\left(\mathcal{I} \times \mathbf{S}^{n} ; \Lambda^{0, p-1}\right)$ such that $L^{\pi} u_{K}-f$ vanishes to infinite order on $K$ and $u_{K} \equiv 0$ for $x<0$.

Proof. Let $\mathcal{T}$ be the tubular neighborhood of $K^{0}$ used in the proof of Lemma 3.6. We set $f=f_{0}+d \rho \wedge \rho^{-1} f_{1}$ in $\mathcal{T} \backslash K^{0}$, with $f_{0} \in \mathcal{C}^{\infty}\left(\mathcal{T} \backslash K^{0} ; \mathbf{E}^{(p)}\right)$ and $f_{1} \in \mathcal{C}^{\infty}\left(\mathcal{T} \backslash K^{0} ; \mathbf{E}^{(p-1)}\right)$ vanishing to infinite order on $K \backslash K^{0}$; moreover $f_{1} \equiv 0$ on $K^{0}$. By applying Lemma 3.6 to $f_{1}$ we get a form $v_{1} \in \mathcal{C}^{\infty}\left(\mathcal{I} \times \mathbf{S}^{n} ; \Lambda^{0, p-1}\right)$ which is a section of $\mathbf{E}^{(p)}$ over $\mathcal{T} \backslash K^{0}$ and has the following properties:

(A) $L_{\rho} v_{1}-\rho^{-1} f_{1}$ vanishes to infinite order on $K$;

(B) $v_{1} \equiv 0$ on $K$;

(C) $\operatorname{supp} v_{1} \subset \mathrm{Cl} \mathcal{T}$. 
When $p=n$ the proof ends here, since $f_{0} \equiv 0$ and $L^{\pi} v_{1}=d \rho \wedge L_{\rho} v_{1}$ (cf. the proof of Lemma 3.4). In the remainder of the proof we assume $p \leq n-1$.

The following is a $\mathcal{C}^{\infty}$ section of $\Lambda^{0, p}$ over $\mathcal{T}$ :

$$
F=f-L^{\pi} v_{1}-d \rho \wedge\left(\rho^{-1} f_{1}-L_{\rho} v_{1}\right)\left(=f_{0}-L_{\Theta} v_{1}\right) ;
$$

it is a section of $\mathbf{E}^{(p)}$ over $\mathcal{T} \backslash K^{0}$ and vanishes to infinite order on $K \backslash K^{0}$; and $L^{\pi} F=(-1)^{p} L^{\pi}\left[\left(\rho^{-1} f_{1}-L_{\rho} v_{1}\right) \wedge d \rho\right]$ vanishes to infinite order on $K^{0}$, i.e., at $\rho=0$.

Next we expand the coefficients of the differential operators $L_{\rho}$ and $L_{\Theta}$ in powers of $\rho$ :

$$
L_{\rho}^{\#}=\partial / \partial \rho+\sum_{a=0}^{\infty} \rho^{a} c_{a} \partial / \partial x, \quad L_{\Theta}^{\#}=d_{\Theta}+\sum_{b=0}^{\infty} \rho^{b} \lambda_{b} \wedge \partial / \partial x .
$$

Since $\lambda_{0}$ is proportional to the pullback of $d_{t} \Phi^{\pi}$ to $K^{0}$ and since each stratum of $K_{x}^{0}$ is contained in a level set of $\Phi^{\pi}(x, \cdot)$, necessarily $\lambda_{0} \equiv 0$. We use now the Taylor expansion in powers of $\rho$ of the section $F$ :

$$
F^{\#}=\sum_{k=0}^{\infty} \rho^{k} F_{k}
$$

we have $L_{\rho}^{\#} F^{\#}=0, L_{\Theta}^{\#} F^{\#}=0$. Explicitly the first one of these equations reads

$$
(k+1) F_{k+1}+\sum_{a+b=k} c_{a} \partial F_{b} / \partial x \equiv 0, \quad \forall k \in \mathbb{Z}_{+} .
$$

On the other hand, since $F$ is a section of $\mathbf{E}^{(p)}$ over $\mathcal{T} \backslash K^{0}, F_{0}$ is equal to the pullback of $F$ to $K^{0}$. When $F_{0} \equiv 0,(3.16)$ entails $F_{k} \equiv 0, \forall k=1,2, \ldots$ : in this case $F$ vanishes to infinite order on $K$, and we can take $u_{K}=v_{1}$.

If $F_{0}$ does not vanish identically we seek a power series $w^{\#}=\sum_{k=0}^{\infty} \rho^{k} w_{k}$ such that every term $\rho^{a} w_{a}$ extends as a $\mathcal{C}^{\infty}$ section of $\Lambda^{0, p-1}$ in $\mathcal{T}$, and such that $L_{\rho}^{\#} w^{\#}=$ $0, L_{\Theta}^{\#} w^{\#}=F^{\#}$. In particular $w^{\#}$ satisfies Equation (3.16) just like $F^{\#}$ :

$$
(k+1) w_{k+1}+\sum_{a+b=k} c_{a} \partial w_{b} / \partial x \equiv 0, \quad \forall k \in \mathbb{Z}_{+} .
$$

As a consequence, once $w_{0}$ is selected the forms $w_{k}$ are determined for all $k \geq 1$. Moreover, multiplying (3.17) by $\rho^{k+1}$ and recalling that $\rho^{a+1} c_{a} \in \mathcal{C}^{\infty}(\mathcal{T})$ shows, by induction on $k$, that every form $\rho^{k} w_{k}$ is smooth in $\mathcal{T}$. On the other hand, pulling back the equation $L_{\Theta}^{\#} w^{\#}=F^{\#}$ to $K^{0}$ leads to

$$
d_{t^{0}} w_{0}=F_{0} \text {. }
$$

It is at this juncture that we avail ourselves of [17, Theorem 9.2]. There is an analytic and subanalytic submanifold $\mathfrak{M}_{0}$ of $\mathbf{S}^{n}$ and a subanalytic isomorphism $\tau_{0}:(0, a) \times \mathfrak{M}_{0} \rightarrow K^{0}$ which commutes with the coordinate projection $\left(x, t^{0}\right) \rightarrow x$. For each $x \in(0, a)$ define $\tau_{0}(x): \mathfrak{M}_{0} \rightarrow K_{x}^{0}$ by the formula $\tau_{0}(x, t)=\left(x, \tau_{0}(x) t\right)$. By hypothesis the pullback $\tau_{0}(x)^{*} F_{0}$ is exact on $\mathfrak{M}_{0}$ for any $x \in(0, a)$. We seek a solution, for each $x \in(0, a)$, of the equation in $\mathfrak{M}_{0}$,

$$
d \tilde{w}_{0}=\tau_{0}(x)^{*} F_{0} .
$$

The $(p-1)$-section $\tilde{w}_{0}$ must be smooth in $(0, a) \times \mathfrak{M}_{0}$ and we need estimates of the derivatives of the coefficients of $\tilde{w}_{0}$ in terms of those of $\tau_{0}(x)^{*} F_{0}$. We can use a subanalytic triangulation of $\mathfrak{M}_{0}[9$, Theorem 2]. We integrate Equation (3.19) with the concomitant estimates, in each simplex of the triangulation (say, by the 
standard homotopy formula), starting with the simplices of highest dimension and descending to those of ever lesser dimension, in the manner, for instance, of [18].

To formulate the estimates it is convenient to use a tubular neighborhood $\mathcal{T}_{0}$ of $\mathfrak{M}_{0}$ in $\mathbf{S}^{n}$ which tapers off at the boundary, defined in the manner of (3.2): $\mathcal{T}_{0}=\bigcup_{\mu \in \mathfrak{M}_{0}} \mathfrak{s}_{\mu}\left(\mathfrak{s}_{\mu}\right.$ is a geodesic ball centered at $\mu$ and perpendicular to $\left.\mathfrak{M}_{0}\right)$; we view $\mathcal{T}_{0}$ as a fibre bundle over $\mathfrak{M}_{0}$ with base projection $\mathfrak{s}_{\mu} \rightarrow \mu$. We can pullback the form $\tilde{w}_{0}$ to $\mathcal{T}_{0}$ under the base projection, and make use of the differential operators in the whole sphere $\mathbf{S}^{n}$. Given any coefficient of $\tilde{w}_{0}, \gamma_{I}$, any integer $j \geq 0$ and any differential operator $P\left(t, \partial_{t}\right)$ in $\mathbf{S}^{n}$, there are constants $C, R>0$ and differential operators $Q_{l}\left(t, \partial_{t}\right)(l=1, \ldots, N)$ such that, for every $x \in(0, a)$,

$$
\begin{aligned}
& \sup _{t \in \mathcal{T}_{0}}\left|\partial_{x}^{j} P\left(t, \partial_{t}\right) \gamma_{I}(x, t)\right| \\
& \quad \leq C \sup _{t \in K_{x}^{0}}\left\{\left[|x|+\operatorname{dist}\left(t, K_{x} \backslash K_{x}^{0}\right)\right]^{-R} \sum_{|J|=p} \sum_{k=0}^{j} \sum_{l=1}^{N}\left|\partial_{x}^{k} Q_{l}\left(t, \partial_{t}\right) F_{J}(x, t)\right|\right\} .
\end{aligned}
$$

Since $F$ vanishes to infinite order on $K \backslash K^{0}$, we may regard $\tilde{w}_{0}$ as a $\mathcal{C}^{\infty}(p-1)$-form in $(0, a) \times \mathcal{T}_{0}$ which does not involve $d x$ and vanishes to infinite order at $x=0$. Now we view $(0, a) \times \mathcal{T}_{0}$ as a fibre bundle over $(0, a) \times \mathfrak{M}_{0}$ with fibres $\{x\} \times \mathfrak{s}_{\mu}$; we also view $\mathcal{T}$ as a fibre bundle over $K^{0}$ (with fibres $\sigma_{x, t^{0}}$ ). Assuming that $\mathcal{T}_{0}$ and $\mathcal{T}$ are sufficiently "thin", we can extend $\tau_{0}$ as a subanalytic fibre bundle isomorphism $\tau$ of $(0, a) \times \mathcal{T}_{0}$ onto a neighborhood of $K^{0}$ which contains $\mathcal{T}$. The pushforward of $\tilde{w}_{0}$ under the map $\tau$ is the sought solution $w_{0}$ of (3.18).

An important consequence of the estimates (3.20) is that $w_{0} \in \mathcal{C}^{\infty}\left(\mathrm{Cl} \mathcal{T} ; \Lambda^{0, p-1}\right)$, by which we mean that the $(p-1)$-form $w_{0}$ and its partial derivatives of all orders extend continuously to the closure of $\mathcal{T}$ in $[0, a) \times \mathbf{S}^{n}$; moreover, they tend to zero as $x \rightarrow+0$. The same will therefore be true of the forms $\rho^{k} w_{k}(k=1,2, \ldots)$ determined by the recurrence relations (3.17) (recall that the functions $\rho^{a+1} c_{a}$ are $\mathcal{C}^{\infty}$ in $\mathrm{Cl} \mathcal{T}$ if $\mathcal{T}$ is sufficiently thin). At this juncture we use once again a cutoff function $\chi \in \mathcal{C}^{\infty}(\mathbb{R}), \chi(\tau)=1$ if $\tau<1, \chi(\tau)=0$ if $\tau>2$. We determine inductively the numbers $\epsilon_{k} \searrow+0$ in such a way that the series $\sum_{k=0}^{\infty} \chi\left(\rho / \epsilon_{k}\right) \rho^{k} w_{k}$ converges in $\mathcal{C}^{\infty}\left(\mathrm{Cl} \mathcal{T} ; \Lambda^{0, p-1}\right)$ to a form $w$ which vanishes to infinite order at $x=0$. By an argument like that used in the proof of Lemma 3.3 to extend the forms $\omega_{k}$ we extend $w$ to $(0, a) \times \mathbf{S}^{n-1}$ as a $\mathcal{C}^{\infty}$ form which vanishes to infinite order as $x \rightarrow+0$; and lastly, we extend $w$ to $\mathcal{I} \times \mathbf{S}^{n-1}$ by setting $w \equiv 0$ if $x<0$. By construction $F-L^{\pi} w$ vanishes to infinite order on $K^{0}$ and therefore also on $K$. The form $u_{K}=v_{1}+w$ satisfies the requirements in Lemma 3.7 since $f-L^{\pi} u_{K}=$ $F-L^{\pi} w+d \rho \wedge\left(\rho^{-1} f_{1}-L_{\rho} v_{1}\right)$.

We are now ready to complete the proof of Lemma 2.1. As before we call $\Sigma_{1}^{(\kappa)}, \ldots, \Sigma_{l_{\kappa}}^{(\kappa)}$ the strata of $\mathcal{A}^{+}$of codimension equal to $\kappa$; and we consider an arbitrary connected component $K$ of $\left(\mathrm{Cl} \Sigma_{1}^{(\kappa)}\right) \cup \cdots \cup\left(\mathrm{Cl} \Sigma_{l_{\kappa}}^{(\kappa)}\right)$. If $\kappa$ is equal to the maximum codimension $\kappa^{+}$of all the strata of $\mathcal{A}^{+}$, then perforce $K \backslash K^{0} \subset \mathcal{F}^{\pi}(0)$. Lemma 3.5 allows us to assume that $f$ vanishes to infinite order on $K \backslash K^{0}$. Now suppose $f$ vanishes to infinite order on every stratum of $\mathcal{A}^{+}$of codimension $>\kappa$, as well as on $\mathcal{F}^{\pi}(0)$. By Lemma 3.7 we can find a form $u_{K} \in \mathcal{C}^{\infty}\left(\mathcal{I} \times \mathbf{S}^{n} ; \Lambda^{0, p-1}\right)$ such that $f-L^{\pi} u_{K}$ vanishes to infinite order on $K$. We may assume that the supports of the forms $u_{K}$ do not intersect, for different connected components $K$ 
of $\left(\mathrm{Cl} \Sigma_{1}^{+(\kappa)}\right) \cup \cdots \cup\left(\mathrm{Cl} \Sigma_{l_{\kappa}}^{+(\kappa)}\right)$. Adding the forms $u_{K}$ yields a form $u^{(\kappa)}$ such that $f-L^{\pi} u^{(\kappa)}$ vanishes to infinite order on $\left(\mathrm{Cl} \Sigma_{1}^{+(\kappa)}\right) \cup \cdots \cup\left(\mathrm{Cl} \Sigma_{l_{\kappa}}^{+(\kappa)}\right)$; moreover, $u^{(\kappa)} \equiv$ 0 if $x<0$. Descending induction on $\kappa$ produces a form $u^{+} \in \mathcal{C}^{\infty}\left(\mathcal{I} \times \mathbf{S}^{n} ; \Lambda^{0, p-1}\right)$ such that $u^{+} \equiv 0$ in the region $x<0$ and that $f-L^{\pi} u^{+}$vanishes to infinite order on $\mathcal{A}^{+}$.

The same argument in the region $x<0$ yields a form $u^{-} \in \mathcal{C}^{\infty}\left(\mathcal{I} \times \mathbf{S}^{n} ; \Lambda^{0, p-1}\right)$ such that $u^{-} \equiv 0$ if $x>0$ and that $f-L^{\pi} u^{-}$vanishes to infinite order on $\mathcal{A}^{-}$; $u_{0}=u^{+}+u^{-}$satisfies the requirements in Lemma 2.1.

\section{Proof of Lemma 2.2. Preparatory estimates}

We refer the reader to the description at the beginning of Section 3. The set of critical values of $Z^{\pi}$ in the rectangle $\mathcal{R}=\{z=x+\iota y \in \mathbb{C} ;|x|<a,|y|<b\}$ (see Lemma 2.1), $\mathcal{R} \cap \mathcal{S}^{\pi}$, consists of $\{0\}$ and of the curves

$$
\begin{aligned}
& 0<x<a, \quad y=\psi_{j}^{+}(x)\left(j=1, \ldots, r^{+}\right) \\
& -a<x<0, \quad y=\psi_{k}^{-}(x)\left(k=1, \ldots, r^{-}\right) .
\end{aligned}
$$

These curves are real-analytic; they are subanalytic sets; $\psi_{j}^{ \pm}(x) \rightarrow 0$ as $x \rightarrow 0$ for all $j=1, \ldots, r^{ \pm}$. If $0<x<a$, then $-b<\psi_{j}^{+}(x)<\psi_{j+1}^{+}(x)<b\left(j=1, \ldots, r^{+}-1\right)$, and if $-a<x<0$, then $-b<\psi_{k}^{-}(x)<\psi_{k+1}^{-}(x)<b\left(k=1, \ldots, r^{-}-1\right)$.

Let $\mathcal{U}$ be an arbitrary connected component of $\mathcal{R} \backslash\left(\mathcal{R} \cap \mathcal{S}^{\pi}\right)$ and $\mathcal{O}$ one of $\left(Z^{\pi}\right)^{-1}(\mathcal{U})$. In other words $\mathcal{U}$ is one of the following sets:

$$
\begin{gathered}
\left\{z \in \mathbb{C} ; 0<x<a, \psi_{j}^{+}(x)<y<\psi_{j+1}^{+}(x)\right\}\left(j=1, \ldots, r^{+}-1\right) ; \\
\left\{z \in \mathbb{C} ;-a<x<0, \psi_{k}^{-}(x)<y<\psi_{k+1}^{-}(x)\right\}\left(k=1, \ldots, r^{-}-1\right) ; \\
\left\{z \in \mathbb{C} ; 0<x<a,-b<y<\psi_{1}^{+}(x)\right\}, \quad\left\{z \in \mathbb{C} ; 0<x<a, \psi_{r^{+}}^{+}(x)<y<b\right\} ; \\
\left\{z \in \mathbb{C} ;-a<x<0,-b<y<\psi_{1}^{-}(x)\right\}, \quad\left\{z \in \mathbb{C} ;-a<x<0, \psi_{r^{-}}^{-}(x)<y<b\right\} .
\end{gathered}
$$

We shall exploit the following obvious facts: $\mathcal{O}$ is a connected component of $\left(Z^{\pi}\right)^{-1}\left(\mathcal{R} \backslash\left(\mathcal{R} \cap \mathcal{S}^{\pi}\right)\right) ; Z^{\pi}(\mathcal{O})=\mathcal{U}$. For each $z \in \mathcal{U}, \mathfrak{L}_{z}=\mathcal{F}^{\pi}(z) \cap \mathcal{O} \subset \mathbf{S}^{n}$ is a compact $\mathcal{C}^{\omega}$ manifold without boundary. As $z$ ranges over $\mathcal{U}$ these manifolds $\mathfrak{L}_{z}$ are diffeomorphic to one another (see below; $\mathcal{O}$ is a fibre bundle over $\mathcal{U}$ whose fibres are the manifolds $\mathfrak{L}_{z}$ ). We endow each submanifold $\mathfrak{L}_{z}$ with the Riemannian metric $g_{(z)}$ induced by the standard metric on $\mathbf{S}^{n}$ and we use the classical Hodge theory in $L^{2}\left(\mathfrak{L}_{z} ; \Lambda^{*}\right)$ defined by means of that metric. To keep this in mind we denote by $(,)_{(z)}$ the inner product on each fibre of the exterior algebra $\Lambda \mathbb{C} T^{*} \mathfrak{L}_{z}$; and by $d_{(z)}, d_{(z)}^{*}$ and $\Delta_{(z)}$ the exterior derivative, its adjoint and the Hodge-Laplacian on the Riemannian manifold $\mathfrak{L}_{z}$.

Unless specified otherwise we shall denote the variable point in $\mathbf{S}^{n}$ by $t$ and we shall regard $\mathfrak{L}_{z}$ as a submanifold of $\mathbf{S}^{n}$. By $\nabla_{t} \Phi^{\pi}$ we shall mean the gradient of $\Phi^{\pi}$ on the sphere. We introduce the quantity

$$
\rho(z)=\operatorname{Min}_{\mathfrak{L}_{z}}\left|\nabla_{t} \Phi^{\pi}(x, t)\right|, \quad z \in \mathcal{U} .
$$

Lemma 4.1. There are constants $c, \mu>0$ such that, for all $z \in \mathcal{U}$,

$$
\rho(z) \geq c\left[\operatorname{dist}\left(z, \mathcal{S}^{\pi}\right)\right]^{\mu} .
$$


Proof. The analyticity of the function $\left|\nabla_{t} \Phi^{\pi}(x, t)\right|^{2}$ allows us to state the following (cf. [13]): there are numbers $c_{1}, \mu>0$ such that, for all $(x, t) \in \mathcal{O}$,

$$
\left|\nabla_{t} \Phi^{\pi}(x, t)\right| \geq c_{1}\left[\operatorname{dist}\left((x, t), \mathcal{N}^{\pi}\right)\right]^{\mu},
$$

where $\mathcal{N}^{\pi}=\left\{(x, t) \in \mathcal{I} \times \mathbf{S}^{n} ;\left|\nabla_{t} \Phi^{\pi}(x, t)\right|=0\right\}$. To each $(x, t) \in \mathcal{O}$ there is $\left(x_{*}, t_{*}\right) \in \mathcal{N}^{\pi}$ such that $\operatorname{dist}\left((x, t), \mathcal{N}^{\pi}\right)=\left[\left|x-x_{*}\right|^{2}+\operatorname{dist}\left(t, t_{*}\right)^{2}\right]^{1 / 2}\left[\operatorname{dist}\left(t, t_{*}\right)\right.$ : geodesic distance between $t$ and $t_{*}$ in $\mathbf{S}^{n}$. Set $y_{*}=\Phi^{\pi}\left(x_{*}, t_{*}\right), z_{*}=x_{*}+\iota y_{*}\left(\in \mathcal{S}^{\pi}\right)$; then

$$
\left|\Phi^{\pi}(x, t)-\Phi^{\pi}\left(x_{*}, t_{*}\right)\right| \leq M\left[\left|x-x_{*}\right|^{2}+\operatorname{dist}\left(t, t_{*}\right)^{2}\right]^{1 / 2},
$$

with $M>0$ independent of $x, t, x_{*}, t_{*}$. If $z=x+\iota \Phi^{\pi}(x, t)$ and if we take (4.2) into account, it follows that $\left|\nabla_{t} \Phi^{\pi}(x, t)\right| \geq c_{1}\left(1+M^{2}\right)^{-\mu / 2}\left|z-z_{*}\right|^{\mu} \geq c_{2}\left[\operatorname{dist}\left(z, \mathcal{S}^{\pi}\right)\right]^{\mu}$ $\left(c_{1}>c_{2}>0\right)$. Taking the minimum of the left-hand side over $t \in \mathfrak{L}_{z}$ yields (4.1).

The forthcoming argument will make use of estimates of the metric $g_{(z)}$ and of its derivatives, both tangential to $\mathfrak{L}_{z}$ and transversal to it, i.e., in the $z$-directions. It is convenient to limit the variation of $z$ to a small open disk $\Delta \Subset \mathcal{U}$ centered at an arbitrary point $z_{0} \in \mathcal{U}$, whose radius $r_{0}$ shall be chosen later.

We shall now reason in a neighborhood of an arbitrary point $O$ in $\mathfrak{L}_{z_{0}}$. After a rotation we may assume that $O$ is the North Pole $(0, \ldots, 0,1)$ of $\mathbf{S}^{n}$ and that the tangent space to $\mathfrak{L}_{z_{0}}$ at $O$ is the affine subspace of $\mathbb{R}^{n+1}$ defined by $t_{n}=0, t_{n+1}=1$. We use the coordinates $t_{1}, \ldots, t_{n}$ in the open Northern Hemisphere $\mathbf{S}_{+}^{n}$ of $\mathbf{S}^{n}$. We shall make systematic use of the notation $t=\left(t^{\prime}, t_{n}\right), t^{\prime}=\left(t_{1}, \ldots, t_{n-1}\right) ; O$ now becomes the origin of $\mathbb{R}^{n}$. For any $z \in \mathcal{U}$ the submanifold $\mathfrak{L}_{z}$ can be defined, in a neighborhood of $O$, by the equation $\Phi^{\pi}(x, t)=y$. At $O$, i.e., when $x=x_{0}$ and $t=0, \partial \Phi^{\pi} / \partial t_{j}=0(1 \leq j<n), \partial \Phi / \partial t_{n}= \pm\left|\nabla_{t} \Phi^{\pi}\right|$. If the radius $r_{0}$ of the disk $\Delta$ and the neighborhood $\omega$ of 0 in $\mathbb{R}^{n}$ are sufficiently small, in $\Delta \times \omega\left(\Subset \mathcal{U} \times \mathbb{R}^{n}\right)$, then $\mathfrak{L}_{z}$ is also defined by an equation $t_{n}=\psi\left(z, t^{\prime}\right)$, with $\psi$ real-valued and real-analytic, $\psi(z, 0)=0, d_{t^{\prime}} \psi(z, 0)=0$. The standard proof of the Implicit Function Theorem shows that we may take $r_{0}=\kappa \rho\left(z_{0}\right)$ and

$$
\omega=\left\{t \in \mathbb{R}^{n} ;\left|t^{\prime}\right|<\kappa \rho\left(z_{0}\right),\left|t_{n}\right|<\kappa \rho^{2}\left(z_{0}\right)\right\}
$$

for some suitably small $\kappa>0$ independent of $z_{0}$ (and a fortiori of the point $O$ ). More precisely, we can select $\kappa>0$ in such a way that, in $\Delta \times \omega$,

$$
\left|\nabla_{t^{\prime}} \Phi^{\pi}\right| \leq M \kappa \rho\left(z_{0}\right), \quad\left|\partial \Phi^{\pi} / \partial t_{n}\right| \geq \frac{1}{2} \rho\left(z_{0}\right),
$$

with $M>0$ also independent of $z_{0}$. Of course,

$$
\begin{gathered}
\Phi^{\pi}(x, t)-y=E(z, t)\left[t_{n}-\psi\left(z, t^{\prime}\right)\right] \quad \text { in } \Delta \times \omega ; \\
\partial \Phi^{\pi} / \partial t_{j}=-E \partial \psi / \partial t_{j}(j=1, \ldots, n-1), \quad \partial \Phi^{\pi} / \partial t_{n}=E \quad \text { in } \Delta \times\left(\mathfrak{L}_{z} \cap \omega\right) .
\end{gathered}
$$

If we write

$$
g_{(z)}=\sum_{i, j=1}^{n-1} g_{i j}\left(z, t^{\prime}\right) d t_{i} \otimes d t_{j}
$$

in $\Delta \times\left(\mathfrak{L}_{z} \cap \omega\right)$, we see that $g_{i j}=\delta_{i j}+\psi_{t_{i}} \psi_{t_{j}}$ or, equivalently,

$$
g_{i j}=\delta_{i j}+\Phi_{t_{i}}^{\pi} \Phi_{t_{j}}^{\pi} /\left(\Phi_{t_{n}}^{\pi}\right)^{2} .
$$


The expression (4.5) defines the $g_{i j}$ as functions of $(x, t)$, and this is how we shall often view them in the sequel. To recover their values on a fixed level set $\mathfrak{L}_{z} \cap \omega$ it suffices to put $\Phi^{\pi}(x, t)=y$ or, which amounts to the same, $t_{n}=\psi\left(z, t^{\prime}\right)$. At any rate, we derive from (4.4) that $\left|g_{i j}-\delta_{i j}\right| \leq 4 M^{2} \kappa^{2}$ in $\Delta \times \omega$, and, as a consequence, given any $\varepsilon>0$ we can choose $\kappa>0$ sufficiently small that

$$
\begin{gathered}
\left|g_{i j}-\delta_{i j}\right| \leq \varepsilon, \\
\left|\operatorname{det}\left(g_{i j}\right)-1\right| \leq \varepsilon, \\
\left|g^{i j}-\delta_{i j}\right| \leq \varepsilon,
\end{gathered}
$$

where the matrix $\left(g^{i j}\right)$ is the inverse of the matrix $\left(g_{i j}\right)$.

This said we consider an exact form $f \in \mathcal{C}^{\infty}\left(\mathfrak{L}_{z} ; \Lambda^{p}\right)$. By the canonical solution of the equation $d_{(z)} v=f$ we shall mean the solution in $L^{2}\left(\mathfrak{L}_{z} ; \Lambda^{p-1}\right)$ which is orthogonal to all closed forms; we denote it by $K_{(z)} f$. It is the unique solution of the pair of equations $d_{(z)} v=f, d_{(z)}^{*} v=0$ in $\mathfrak{L}_{z}$. Equivalently, $K_{(z)} f$ is the (unique) solution in $L^{2}\left(\mathfrak{L}_{z} ; \Lambda^{p-1}\right)$ of the Hodge-Laplace equation $\Delta_{(z)} v=d_{(z)}^{*} f$ orthogonal to all harmonic $(p-1)$-forms. [Among other things this proves that $K_{(z)} f$ is smooth.]

We are going to estimate the $L^{2}$ norms of the (tangential) derivatives of the canonical solution $K_{\left(z_{0}\right)} f$ in terms of the $L^{2}$ norms of the derivatives of the exact form $f \in \mathcal{C}^{\infty}\left(\mathfrak{L}_{z_{0}} ; \Lambda^{p}\right)$, with constants that depend on $z_{0}$ only through $\operatorname{dist}\left(z_{0}, \mathcal{S}^{\pi}\right)$. This will be possible thanks to the appropriate choice of the parameters $N, r, A$ in Lemma A.1 in which we take $\mathcal{M}=\mathfrak{L}_{z_{0}}$ (and therefore $m=n-1$ ).

Lemma 4.2. There are positive constants $C^{\prime}, \mu^{\prime}$ independent of $z_{0}$ such that, for any exact form $f \in \mathcal{C}^{\infty}\left(\mathfrak{L}_{z_{0}} ; \Lambda^{p}\right)$,

$$
\left\|K_{\left(z_{0}\right)} f\right\|_{L^{2}} \leq C^{\prime}\left[\operatorname{dist}\left(z_{0}, \mathcal{S}^{\pi}\right)\right]^{-\mu^{\prime}}\|f\|_{L^{2}}
$$

Proof. We adapt part of the argument in [2]. By (4.4) we know that, by choosing the number $\kappa$ suitably small, we can render the exponential map $T_{O} \mathfrak{L}_{z_{0}} \rightarrow \mathfrak{L}_{z_{0}} \cap \omega$ as close as we wish to the map $t^{\prime} \rightarrow\left(t^{\prime}, \psi\left(z_{0}, t^{\prime}\right)\right)$. The size of $\omega$ shows that, in applying Lemma A.1 to $\mathcal{M}=\mathfrak{L}_{z_{0}}$, we have the right to take $r=\kappa_{1} \rho\left(z_{0}\right)$ with $\kappa_{1}>0$ suitably small and independent of $z_{0}$. And the constant $A$ in Lemma A.1 can be taken $\leq 2$. Finally, by a general result of $[8]$ (Corollary 2.10, p. 93) according to which Vol $\mathfrak{L}_{z}$ is bounded independently of $z \in \mathcal{R} \backslash \mathcal{R} \cap \mathcal{S}^{\pi}$, our choice of $r$ allows us to apply Lemma A.1 with $N \leq C^{\prime} / \rho\left(z_{0}\right)^{n-1}$ and $C^{\prime}>0$ also independent of $z_{0}$ (cf. [2, Lemma 5]).

All this goes to show that (A.4) specializes, in our situation, to an estimate

$$
\left\|K_{\left(z_{0}\right)} f\right\|_{L^{2}} \leq C^{\prime \prime} \rho\left(z_{0}\right)^{4 p+1-4 n p}\|f\|_{L^{2}} .
$$

Combining this inequality with (4.1) yields (4.9).

Corollary 4.3. Let the constants $C^{\prime}, \mu^{\prime}$ be as in Lemma 4.2. Then, for any exact form $f \in \mathcal{C}^{\infty}\left(\mathfrak{L}_{0} ; \Lambda^{p}\right)$ and for any integer $k \geq 0$,

$$
\left\|\Delta_{\left(z_{0}\right)}^{k} K_{\left(z_{0}\right)} f\right\|_{L^{2}} \leq C^{\prime}\left[\operatorname{dist}\left(z_{0}, \mathcal{S}^{\pi}\right)\right]^{-\mu^{\prime}}\left\|\Delta_{\left(z_{0}\right)}^{k} f\right\|_{L^{2}}
$$

Proof. Apply (4.9) with $\Delta_{\left(z_{0}\right)}^{k} f$ instead of $f$, and take into account the commutation relation $\Delta_{(z)} K_{(z)}=K_{(z)} \Delta_{(z)}$. 
The estimate (4.10) enables us to estimate the derivatives of $K_{\left(z_{0}\right)} f$ in the directions tangential to $\mathfrak{L}_{z_{0}}$. The Hodge-Laplacian acting on $q$-forms is given by

$$
\Delta_{\left(z_{0}\right)}=-\Delta_{0}+R
$$

([21]), where $\Delta_{0}$ is the Laplace-Beltrami operator in $\mathfrak{L}_{z_{0}}$, acting coefficientwise, and $R$ is a multiplication operator related to the Riemann tensor. In a local chart such as $\left(\omega \cap \mathfrak{L}_{z_{0}}, t_{1}, \ldots, t_{n-1}\right)$ [see (4.3)] its entries are polynomials with respect to the $g_{i j}$ and their derivatives of order $\leq 2$. It follows from (4.4) and (4.5) that an estimate $|R| \leq B_{0} \rho\left(z_{0}\right)^{-\mu_{0}}$ is valid on $\mathfrak{L}_{z_{0}}$, with constants $B_{0}, \mu_{0}>0$ independent of $z_{0}$. More generally we have, in the local chart $\left(\omega \cap \mathfrak{L}_{z_{0}}, t_{1}, \ldots, t_{n-1}\right)$,

$$
\left|\left(\partial / \partial t^{\prime}\right)^{\alpha} R\right| \leq B_{k} \rho\left(z_{0}\right)^{-\mu_{k}} \quad \text { if } \alpha \in \mathbb{Z}_{+}^{n-1},|\alpha| \leq k,
$$

with constants $B_{k}, \mu_{k}>0$ independent of $z_{0}$.

Select a cutoff function $\chi \in \mathcal{C}_{c}^{\infty}\left(\omega \cap \mathfrak{L}_{z_{0}}\right), \chi \equiv 1$ in a neighborhood of $O \in \omega$, such that $\operatorname{dist}\left(\operatorname{supp} \chi, \mathbf{S}^{n} \backslash \omega\right) \geq \kappa^{\prime} \rho\left(z_{0}\right)$ and that, for all $k \in \mathbb{Z}_{+}$,

$$
\left|\left(\partial / \partial t^{\prime}\right)^{\alpha} \chi\right| \leq B_{k}^{\prime} \rho\left(z_{0}\right)^{-k} \text { if } \alpha \in \mathbb{Z}_{+}^{n-1},|\alpha| \leq k .
$$

Both constants $\kappa^{\prime}$ and $B_{k}^{\prime}$ shall be independent of $z_{0}$.

Let $(,)_{L^{2}}$ stand for the inner product in $L^{2}\left(\mathfrak{L}_{z_{0}}\right)$. We recall that if $u, v \in L^{2}\left(\mathfrak{L}_{z_{0}}\right)$ and if $\operatorname{supp}(u \bar{v}) \subset \omega \cap \mathfrak{L}_{z_{0}}$, then

$$
(u, v)_{L^{2}}=\int_{\mathbb{R}^{n-1}} u \bar{v}\left[\operatorname{det}\left(g_{i j}\right)\right]^{1 / 2} d t_{1} \cdots d t_{n-1} .
$$

We avail ourselves of $(4.5),(4.6),(4.7)$, (4.8). If $u \in \mathcal{C}^{\infty}\left(\mathfrak{L}_{z_{0}} ; \Lambda^{p-1}\right)$, in $\omega \cap \mathfrak{L}_{z_{0}}$, we may write $u=\sum_{|I|=p-1} u_{I} d t_{I}$ with multi-indices $I$ that consist of integers $i<n$. We begin by observing that to each integer $k \geq 0$ there is a constant $B_{k}^{\prime \prime}>0$ (independent of $z_{0}$ and of $u$ ) such that

$$
\sum_{|\alpha|=k}\left\|\left(\partial / \partial t^{\prime}\right)^{\alpha}\left(\chi u_{I}\right)\right\|_{L^{2}}^{2} \leq B_{k}^{\prime \prime} \sum_{l=0}^{k}\left|\left(\Delta_{t^{\prime}}^{l}\left(\chi u_{I}\right), \chi u_{I}\right)_{L^{2}}\right|,
$$

where we have used the notation $\Delta_{t^{\prime}}=\sum_{j=1}^{n-1}\left(\partial / \partial t_{j}\right)^{2}$.

Next we exploit the fact that the expression of the Laplace-Beltrami operator in the coordinates $t_{1}, \ldots, t_{n-1}$ is close to $\Delta_{t^{\prime}}$. More precisely, if the constant $\kappa$ is sufficiently small there will be $M>0$, independent of $z_{0}$ and of $u$, such that

$$
\begin{aligned}
& \sum_{l=0}^{k}\left|\left(\Delta_{t^{\prime}}^{l}\left(\chi u_{I}\right), \chi u_{I}\right)_{L^{2}}-\left(\Delta_{0}^{l}\left(\chi u_{I}\right), \chi u_{I}\right)_{L^{2}}\right| \\
& \quad \leq \frac{1}{2} B_{k}^{\prime \prime-1} \sum_{|\alpha|=k}\left\|\left(\partial / \partial t^{\prime}\right)^{\alpha}\left(\chi u_{I}\right)\right\|_{L^{2}}^{2}+M \rho\left(z_{0}\right)^{-2 k} \sum_{|\alpha|<k}\left\|\left(\partial / \partial t^{\prime}\right)^{\alpha}\left(\chi u_{I}\right)\right\|_{L^{2}}^{2} .
\end{aligned}
$$

If we recall that

$$
\Delta_{0}^{l}(\chi u)=\sum_{|I|=p-1} \Delta_{0}^{l}\left(\chi u_{I}\right) d t_{I}
$$


we see that

$$
\begin{aligned}
\sum_{|I|=p-1} \sum_{|\alpha|=k}\left\|\left(\partial / \partial t^{\prime}\right)^{\alpha}\left(\chi u_{I}\right)\right\|_{L^{2}}^{2} \leq & 2 B_{k}^{\prime \prime} \sum_{l=0}^{k} \mid\left(\Delta_{0}^{l}(\chi u), \chi u\right)_{L^{2} \mid} \\
& +2 M \rho\left(z_{0}\right)^{-2 k} \sum_{|I|=p-1} \sum_{|\alpha|<k}\left\|\left(\partial / \partial t^{\prime}\right)^{\alpha}\left(\chi u_{I}\right)\right\|_{L^{2}}^{2} .
\end{aligned}
$$

By taking Formula (4.11) and the estimates (4.12) into account, we obtain, for suitably large constants $C_{k}$ and $\mu_{k}$ (independent of $z_{0}$ and of $u$ ),

$$
\begin{aligned}
\sum_{|I|=p-1} \sum_{|\alpha|=k}\left\|\left(\partial / \partial t^{\prime}\right)^{\alpha}\left(\chi u_{I}\right)\right\|_{L^{2}}^{2} & \leq 2 B_{k}^{\prime \prime} \sum_{l=0}^{k}\left(\Delta^{l}(\chi u), \chi u\right)_{L^{2}} \\
& +C_{k} \rho\left(z_{0}\right)^{-\mu_{k}} \sum_{|I|=p-1} \sum_{|\alpha|<k}\left\|\left(\partial / \partial t^{\prime}\right)^{\alpha}\left(\chi u_{I}\right)\right\|_{L^{2}}^{2} .
\end{aligned}
$$

Now select a function $\chi_{1} \in \mathcal{C}_{c}^{\infty}\left(\omega \cap \mathfrak{L}_{z_{0}}\right), \chi_{1} \equiv 1$ in a neighborhood of supp $\chi$, $0 \leq \chi_{1} \leq 1$ everywhere, and satisfying estimates similar to (4.13) with larger constants $A_{k}$. Due to the inequality

$$
\begin{aligned}
\sum_{l=0}^{k}\left(\Delta^{l}(\chi u), \chi u\right)_{L^{2}} \leq & \left|\sum_{l=0}^{k}\left(\chi \Delta^{l} u, \chi u\right)_{L^{2}}\right| \\
& +M \rho\left(z_{0}\right)^{-2 k} \sum_{|I|=p-1} \sum_{|\alpha|<k}\left\|\left(\partial / \partial t^{\prime}\right)^{\alpha}\left(\chi_{1} u_{I}\right)\right\|_{L^{2}}^{2}
\end{aligned}
$$

we obtain

$$
\begin{aligned}
\sum_{|I|=p-1} \sum_{|\alpha|=k}\left\|\left(\partial / \partial t^{\prime}\right)^{\alpha}\left(\chi u_{I}\right)\right\|_{L^{2}}^{2} \leq & 2 B_{k}^{\prime \prime}\left|\sum_{l=0}^{k}\left(\chi \Delta^{l} u, \chi u\right)_{L^{2}}\right| \\
& +C_{k}^{\prime} \rho\left(z_{0}\right)^{-\mu_{k}^{\prime}} \sum_{|I|=p-1} \sum_{|\alpha|<k}\left\|\left(\partial / \partial t^{\prime}\right)^{\alpha}\left(\chi_{1} u_{I}\right)\right\|_{L^{2}}^{2},
\end{aligned}
$$

where the positive constants $C_{k}^{\prime}, \mu_{k}^{\prime}$ are independent of $z_{0}$ and $u$.

We apply this to $u=K_{\left(z_{0}\right)} f$. By combining the preceding inequality with $(4.10)$ and by taking (4.1) into account once again we get

$$
\begin{aligned}
\sum_{|\alpha|=k}\left\|\left(\partial / \partial t^{\prime}\right)^{\alpha}\left(\chi K_{\left(z_{0}\right)} f\right)\right\|_{L^{2}}^{2} & \leq 2 B_{k}^{\prime \prime} C^{\prime}\left[\operatorname{dist}\left(z_{0}, \mathcal{S}^{\pi}\right)\right]^{-\mu^{\prime}}\left\|\Delta_{\left(z_{0}\right)}^{k} f\right\|_{L^{2}}^{2} \\
& +C_{k}^{\prime}\left[\operatorname{dist}\left(z_{0}, \mathcal{S}^{\pi}\right)\right]^{-\mu_{k}^{\prime}} \sum_{|\alpha|<k}\left\|\left(\partial / \partial t^{\prime}\right)^{\alpha}\left(\chi_{1} K_{\left(z_{0}\right)} f\right)\right\|_{L^{2}}^{2}
\end{aligned}
$$

Since the cutoff function $\chi_{1}$ is of the same type as $\chi$, a simple induction argument (and an increase of the constants $C_{k}^{\prime}, \mu_{k}^{\prime}$ ) yields the inequality

$$
\sum_{|\alpha|=k}\left\|\left(\partial / \partial t^{\prime}\right)^{\alpha}\left(\chi K_{\left(z_{0}\right)} f\right)\right\|_{L^{2}}^{2} \leq C_{k}^{\prime}\left[\operatorname{dist}\left(z_{0}, \mathcal{S}^{\pi}\right)\right]^{-\mu_{k}^{\prime}} \sum_{l=0}^{k}\left\|\Delta_{\left(z_{0}\right)}^{l} f\right\|_{L^{2}}^{2}
$$

Applying a rough version of the Sobolev Lemma to (4.15) allows us to conclude that to every integer $k \geq 0$ there are positive constants $C_{k}^{\prime \prime}, \mu_{k}^{\prime \prime}$ (independent of $z_{0}$ ) 
such that the following is true, for any exact form $f \in \mathcal{C}^{\infty}\left(\mathfrak{L}_{z_{0}} ; \Lambda^{p}\right)$ :

$$
\sum_{|\alpha| \leq k}\left\|\left(\partial / \partial t^{\prime}\right)^{\alpha}\left(\chi K_{\left(z_{0}\right)} f\right)\right\|_{L^{\infty}} \leq C_{k}^{\prime \prime}\left[\operatorname{dist}\left(z_{0}, \mathcal{S}^{\pi}\right)\right]^{-\mu_{k}^{\prime \prime}} \sum_{l=0}^{n+k}\left\|\Delta_{\left(z_{0}\right)}^{l} f\right\|_{L^{2}}
$$

We must emphasize the fact that in all the preceding estimates the operators $\left(\partial / \partial t^{\prime}\right)^{\alpha}$ act tangentially to $\mathfrak{L}_{z_{0}}$. In other words, for each $j=1, \ldots, n-1, \partial / \partial t_{j}$ should be interpreted as the pushforward of the vector field $\partial / \partial t_{j}$ in $\mathbb{R}^{n-1}$ under the map $t^{\prime} \rightarrow\left(t^{\prime}, \psi\left(z_{0}, t^{\prime}\right)\right)$, i.e., as the vector field

$$
\partial / \partial t_{j}+\left(\partial \psi / \partial t_{j}\right) \partial / \partial t_{n}=\partial / \partial t_{j}-\left(\partial \Phi^{\pi} / \partial t_{n}\right)^{-1}\left(\partial \Phi^{\pi} / \partial t_{j}\right) \partial / \partial t_{n} .
$$

If we want to free the estimates (4.16) from the choice of special tangential coordinates (such as $t_{1}, \ldots, t_{n-1}$ in $\omega \cap \mathfrak{L}_{z_{0}}$ ) we must use vector fields such as

$$
\left(\partial \Phi^{\pi} / \partial t_{i}\right) \partial / \partial t_{j}-\left(\partial \Phi^{\pi} / \partial t_{j}\right) \partial / \partial t_{i} \quad(1 \leq i<j \leq n)
$$

which span the tangent bundle of each level manifold $\mathfrak{L}_{z}$. Actually it is convenient to normalize those vector fields and use

$$
X_{i j}=\left|\nabla \Phi^{\pi}\right|^{-1}\left[\left(\partial \Phi^{\pi} / \partial t_{i}\right) \partial / \partial t_{j}-\left(\partial \Phi^{\pi} / \partial t_{j}\right) \partial / \partial t_{i}\right] \quad(1 \leq i<j \leq n) .
$$

An easy induction argument and repeated use of the Lojasiewicz inequality (4.2) show that the estimates (4.16) allow us to conclude the following:

Lemma 4.4. To every integer $k \geq 0$ there are positive constants $C_{k}^{\prime \prime \prime}, \mu_{k}^{\prime \prime \prime}$ such that the following is true, for any $z \in \mathcal{U}$, any sequence of $k$ vector fields $X_{i_{1} j_{1}}, \ldots, X_{i_{k} j_{k}}$ and any exact form $f \in \mathcal{C}^{\infty}\left(\mathfrak{L}_{z} ; \Lambda^{p}\right)$ :

$$
\left\|X_{i_{1} j_{1}} \cdots X_{i_{k} j_{k}} K_{(z)} f\right\|_{L^{\infty}} \leq C_{k}^{\prime \prime \prime}\left[\operatorname{dist}\left(z, \mathcal{S}^{\pi}\right)\right]^{-\mu_{k}^{\prime \prime \prime}} \sum_{l=0}^{n+k}\left\|\Delta_{(z)}^{l} f\right\|_{L^{2}},
$$

with the $L^{p}$ norms computed over $\mathfrak{L}_{z}$.

\section{End of the PRoOf of Lemma 2.2}

We now move to a set-up in which variation "transversal" to the manifolds $\mathfrak{L}_{z}$ must be taken into account: the coefficients of the forms under study will be defined in the open set $\mathcal{O}$ and vary with $z \in \mathcal{U}$; we shall seek estimates of their derivatives both with respect to $t$ and to $z$. Recall that $\mathcal{U}$ is a connected component of $\mathcal{R} \backslash\left(\mathcal{R} \cap \mathcal{S}^{\pi}\right)$ and that $\mathcal{O} \subset U^{\pi}=\left(Z^{\pi}\right)^{-1}(\mathcal{R})$ is one of $\left(Z^{\pi}\right)^{-1}(\mathcal{U})$. To establish the desired estimates on the level set $\mathfrak{L}_{z_{0}}$ we let $z$ vary in the disk $\Delta=\left\{z \in \mathbb{C} ;\left|z-z_{0}\right|<r_{0}\right\} \Subset \mathcal{U}$.

We shall deal with a form $F \in \mathcal{C}_{*}^{\infty}\left(U^{\pi} ; \Lambda^{0, p}\right)$ whose pullback to every regular fibre of $Z^{\pi}$ is exact (cf. Lemma 2.2). For each $z \in \mathcal{U}$ we denote by $F_{z} \in \mathcal{C}^{\infty}\left(\mathfrak{L}_{z} ; \Lambda^{p}\right)$ the pullback of $F$ to $\mathfrak{L}_{z}=\left\{(x, t) \in \mathcal{O} ; x+\iota \Phi^{\pi}(x, t)=z\right\}$. We call $v$ the $(p-1)$-form in $\mathcal{O}$ whose pullback to each $\mathfrak{L}_{z}$ is equal to the canonical solution $v_{z}=K_{(z)} F_{z}$ and whose contraction, when $p \geq 2$, with either $\partial / \partial x$ or the gradient of $\Phi^{\pi}$ on the sphere $\mathbf{S}^{n}$, vanishes identically. By using local trivializations of the fibre bundle $\mathcal{O} \rightarrow \mathcal{U}$ it is checked at once that $v$ is smooth. In order to prove that $v$ vanishes to infinite order on the boundary of $\mathcal{O}$ we need estimates of the derivatives of the coefficients of $v_{z}$ both in the tangential and transversal directions, extending (4.17).

Let us outline our approach to such estimates. To each $z \in \Delta$ we associate a diffeomorphism $J(z): \mathfrak{L}_{z_{0}} \rightarrow \mathfrak{L}_{z}$ such that $J\left(z_{0}\right)=$ Identity of $\mathfrak{L}_{z_{0}}$, with the following properties. In the product space $\mathcal{U} \times \mathbf{S}^{n}$ let $\Sigma$ be the hypersurface defined 
by the equation $y=\Phi^{\pi}(x, t)$; $\operatorname{dim} \Sigma=n+1$. Note that $\mathcal{O}$ can be regarded as an open subset of $\Sigma$ and that the level sets $\mathfrak{L}_{z}$ have codimension two with respect to $\Sigma$. [In the sequel we continue to think of $\mathfrak{L}_{z}$ as a subset of $\mathbf{S}^{n}$ rather than one of $\mathcal{U} \times \mathbf{S}^{n}$. Unless otherwise specified the variable point in $\mathbf{S}^{n}$ will be called $t$ and we shall write $t \in \mathfrak{L}_{z}$ rather than $(z, t) \in \mathfrak{L}_{z}$.] Consider then a ray $\left[0, \sigma_{0}\right] \ni \sigma \rightarrow z(\theta, \sigma)=z_{0}+\sigma e^{\iota \theta} \in \mathcal{U}\left(0<\sigma_{0}<r_{0}=\right.$ radius of $\left.\Delta\right)$. The map $\left[0, \sigma_{0}\right] \times \mathfrak{L}_{z_{0}} \ni(\theta, \sigma, \wp) \rightarrow t(\theta, \sigma, \wp)=J(z(\theta, \sigma)) \wp$ will represent the flow of a vector field $\vec{\nu}$ tangent to $\Sigma$ [meaning that $\Phi^{\pi}(x(\theta, \sigma), t(\theta, \sigma, \wp))=y(\theta, \sigma)$ ] whose direction is unambiguously defined: at each point $(z, t) \in \Sigma(z \in \Delta), \vec{\nu}$ will be perpendicular to $\mathfrak{L}_{z}$; the projection $(z, t) \rightarrow z$ will map $\vec{\nu}$ into the ray $z(\theta, \sigma)$. Let us say right away that it will suffice to deal with horizontal and vertical rays, i.e., with $\theta=0, \pi$ and $\theta= \pm \pi / 2$. For this reason we shall not mention the parameter $\theta$ any more; we write $z(\sigma)$ rather than $z(\theta, \sigma)$, and $t(\sigma, \wp)$ rather than $t(\theta, \sigma, \wp)$.

Before looking more closely at the diffeomorphism $J(z)$ let us show how we plan to use it. If $J(z)^{*}$ denotes the pullback of differential forms from $\mathfrak{L}_{z}$ to $\mathfrak{L}_{z_{0}}$ under the map $J(z)$, then we have, in $\mathfrak{L}_{z_{0}}$,

$$
d_{\left(z_{0}\right)}\left(J(z)^{*} v_{z}\right)=J(z)^{*} F_{z} .
$$

Put $z=z(\sigma), \sigma>0$; let $(\partial / \partial \sigma)^{l}(l=1,2, \ldots)$ act on both sides of $(5.1)$ and put $\sigma=0$ in the result. We get

$$
d_{\left(z_{0}\right)}\left(\left.(\partial / \partial \sigma)^{l}\left[J(z(\sigma))^{*} v_{z(\sigma)}\right]\right|_{\sigma=0}\right)=\left.(\partial / \partial \sigma)^{l}\left[J(z(\sigma))^{*} F_{z(\sigma)}\right]\right|_{\sigma=0} .
$$

Later in this section we shall establish "good" estimates for the derivatives, both tangential and transversal to $\mathfrak{L}_{z_{0}}$, of $J(z(\sigma))^{*}$ (at $\sigma=0$ ). This will yield good tangential estimates for the right-hand side of (5.2). Unfortunately, when $l \geq 1$, this does not enable us to apply Lemma 4.4 to $\left.(\partial / \partial \sigma)^{l}\left[J(z(\sigma))^{*} v_{z(\sigma)}\right]\right|_{\sigma=0}$, since we don't know whether this $(p-1)$-form is the canonical solution of the equation (5.2).

In order to circumvent this difficulty we are going to introduce a form $\alpha^{(l)} \in$ $\mathcal{C}^{\infty}\left(\mathfrak{L}_{z_{0}} ; \Lambda^{p-1}\right)$ orthogonal in $L^{2}\left(\mathfrak{L}_{z_{0}} ; \Lambda^{p-1}\right)$ to all closed forms and such that the tangential derivatives of

$$
\beta^{(l)}=\alpha^{(l)}-\left.(\partial / \partial \sigma)^{l}\left[J(z(\sigma))^{*} v_{z(\sigma)}\right]\right|_{\sigma=0}
$$

can be easily estimated, using Lemma 4.4 and induction on $l=0,1, \ldots$ Then we shall be able to apply Lemma 4.4 to the equation

$$
d_{\left(z_{0}\right)} \alpha^{(l)}=\left.(\partial / \partial \sigma)^{l}\left[J(z(\sigma))^{*} F_{z(\sigma)}\right]\right|_{\sigma=0}+d_{\left(z_{0}\right)} \beta^{(l)}
$$

and obtain good estimates of the derivatives of $\alpha^{(l)}$, and thereby of those in $\left.(\partial / \partial \sigma)^{l}\left[J(z(\sigma))^{*} v_{z(\sigma)}\right]\right|_{\sigma=0}$

We have, for any closed form $\varphi$ on $\mathfrak{L}_{z_{0}}$ and any $z \in \mathcal{U}$ near $z_{0}$,

$$
\int_{\mathfrak{L}_{z}}\left(v_{z},\left(J(z)^{-1}\right)^{*} \varphi\right)_{(z)} d V(z)=0
$$

with $d V(z) \in \mathcal{C}^{\infty}\left(\mathfrak{L}_{z} ; \Lambda^{n-1}\right)$ the volume element in $\mathfrak{L}_{z}$. In the integral at the left we make the change of variables $t=J(z) \tau \rightarrow \tau$. In doing this it is convenient to cover the submanifold $\mathfrak{L}_{z_{0}}$ with coordinate patches of the kind (4.3) [in which (4.4) to (4.8) hold]. Thus we restrict the variation of $z$ to the disk $\Delta$ and assume that $\operatorname{supp} \varphi \Subset \omega$. The generic point $\wp \in \mathfrak{L}_{z_{0}} \cap \omega$ is determined by its coordinates $t_{1}, \ldots, t_{n-1}$. To avoid confusion we shall write $t_{j}(\wp)=\tau_{j}, \tau^{\prime}=\left(\tau_{1}, \ldots, \tau_{n-1}\right)$. It is convenient to 
identify $\wp$ to $\tau=\left(\tau^{\prime}, \tau_{n}\right)$ with $\tau_{n}=\psi\left(z_{0}, \tau^{\prime}\right)$. We write $J(z) \tau=t(z, \tau)$; we have $t\left(z_{0}, \tau\right)=\tau$. We assume throughout that $t(z, \tau) \in \omega$ for any $z \in \Delta$ and $\tau \in \mathfrak{L}_{z_{0}} \cap \omega$.

With the $g_{i j}$ given by (4.5) and $t=\left(t^{\prime}, \psi\left(z, t^{\prime}\right)\right)$, we have, in $\Delta \times \omega$,

$$
\begin{aligned}
d V(z) & =\left[\operatorname{det}\left(g_{i j}(x, t)\right)\right]^{\frac{1}{2}} d t_{1} \cdots d t_{n-1} \\
& =\left[\operatorname{det}\left(g_{i j}(x, J(z) \tau)\right)\right]^{\frac{1}{2}} d[J(z) \tau]_{1} \cdots d[J(z) \tau]_{n-1} \\
& =\left[\operatorname{det}\left(g_{i j}(x, J(z) \tau)\right)\right]^{\frac{1}{2}}\left|\frac{D t(z, \tau)}{D \tau}\right| d \tau_{1} \cdots d \tau_{n-1}=\mathcal{Q}(z, \tau) d V\left(z_{0}\right),
\end{aligned}
$$

where

$$
\mathcal{Q}(z, \tau)=\left[\operatorname{det}\left(g_{i j}(x, J(z) \tau)\right)\right]^{\frac{1}{2}}\left|\frac{D t(z, \tau)}{D \tau}\right|\left[\operatorname{det}\left(g_{i j}\left(x_{0}, \tau\right)\right)\right]^{-\frac{1}{2}} .
$$

Here $\tau_{n}=\psi\left(z_{0}, \tau^{\prime}\right)$. Thus the integral at the left in (5.4) is equal to

$$
\int_{\mathfrak{L}_{z_{0}}}\left(v_{z},\left(J(z)^{-1}\right)^{*} \varphi\right)_{(z)} \mathcal{Q}(z, \tau) d V\left(z_{0}\right),
$$

where $\left(v_{z},\left(J(z)^{-1}\right)^{*} \varphi\right)_{(z)}$ is regarded as a function of $\tau \in \mathfrak{L}_{z_{0}}$. We must take a closer look at the integrand.

Let us write

$$
w=J(z)^{*} v_{z}=\sum_{|I|=p-1} w_{I}(z, \tau) d \tau_{I} \in \mathcal{C}^{\infty}\left(\mathfrak{L}_{z_{0}} ; \Lambda^{p-1}\right) ;
$$

thus

$$
\begin{aligned}
v_{z} & =\left(J(z)^{-1}\right)^{*} w=\sum_{|I|=p-1} w_{I}\left(z, J(z)^{-1} t\right) d\left[J(z)^{-1} t\right]_{I} \\
& =\sum_{|H|=|I|=p-1} w_{H}\left(z, J(z)^{-1} t\right) \Gamma_{H, I}(z, t) d t_{I},
\end{aligned}
$$

where $\Gamma_{H, I}(z, t)$ is the appropriate minor of the Jacobian determinant of $J(z)^{-1}$. Likewise

$$
\left(J(z)^{-1}\right)^{*} \varphi=\sum_{|J|=|K|=p-1} \varphi_{K}\left(J(z)^{-1} t\right) \Gamma_{K, J}(z, t) d t_{J} .
$$

Now consider any two forms $\alpha, \beta \in \mathcal{C}^{\infty}\left(\omega ; \Lambda^{p-1}\right)$ :

$$
\alpha=\sum_{|I|=p-1} \alpha_{I}(t) d t_{I}, \quad \beta=\sum_{|J|=p-1} \beta_{J}(t) d t_{J},
$$

where $I, J$ are ordered multi-indices of integers between 1 and $n-1$. There is a matrix with smooth entries $A_{I, J}(z, t)$ in $\Delta \times \omega$ such that

$$
(\alpha, \beta)_{(z)}=\sum_{|I|=|J|=p-1} A_{I, J}(z, t) \alpha_{I}(t) \overline{\beta_{J}(t)} .
$$

Later on we shall give explicit expressions of the entries $A_{I, J}$. Right now we derive the following from what precedes:

$$
\begin{aligned}
& \left(v_{z},\left(J(z)^{-1}\right)^{*} \varphi\right)_{(z)} \\
& \quad=\sum_{|H|=|I|=|J|=|K|=p-1} A_{I, J}(z, t) \Gamma_{K, J}(z, t) \Gamma_{H, I}(z, t) w_{H}\left(z, J(z)^{-1} t\right) \overline{\varphi_{K}\left(J(z)^{-1} t\right)} .
\end{aligned}
$$


In this expression we put $t=J(z) \tau$; we get

$$
\begin{aligned}
& \left(v_{z},\left(J(z)^{-1}\right)^{*} \varphi\right)_{(z)} \\
& \quad=\sum_{|H|=|I|=|J|=|K|=p-1} A_{I, J}(z, J(z) \tau) \Gamma_{K, J}(z, J(z) \tau) \Gamma_{H, I}(z, J(z) \tau) w_{H}(z, \tau) \overline{\varphi_{K}(\tau)} .
\end{aligned}
$$

Now let $B_{I, J}$ denote the entries of the inverse of the matrix $\left(A_{I, J}\right)$. We define the form in $\mathfrak{L}_{z_{0}} \cap \omega$,

$$
\tilde{v}(z)=\sum_{|I|=p-1} \tilde{v}_{I}(z, \tau) d \tau_{I}
$$

with

$$
\begin{array}{r}
\tilde{v}_{I}(z, \tau)=\mathcal{Q}(z, \tau) \sum_{|H|=\left|H^{\prime}\right|=|J|=|K|=p-1} B_{I, K}\left(z_{0}, \tau\right) A_{H^{\prime}, J}(z, J(z) \tau) \\
\cdot \Gamma_{K, J}(z, J(z) \tau) \Gamma_{H, H^{\prime}}(z, J(z) \tau) w_{H}(z, \tau) .
\end{array}
$$

We have

$$
\mathcal{Q}(z, \tau)\left(v_{z},\left(J(z)^{-1}\right)^{*} \varphi\right)_{(z)}=\sum_{|I|=|J|=p-1} A_{I, J}\left(z_{0}, \tau\right) \tilde{v}_{I}(z, \tau) \overline{\varphi_{J}(\tau)}=(\tilde{v}, \varphi)_{\left(z_{0}\right)}
$$

In passing note that $\tilde{v}\left(z_{0}\right)=v_{z_{0}}$.

We may write $\tilde{v}(z)=M(z) w$, with $M(z)$ a "multiplication operator" acting on $(p-1)$-forms in $\omega \cap \mathfrak{L}_{z_{0}}$ : if $\chi=\sum_{|H|=p-1} \chi_{H}(\tau) d \tau_{H} \in \mathcal{C}^{\infty}\left(\omega \cap \mathfrak{L}_{z_{0}} ; \Lambda^{p-1}\right)$,

$$
M(z) \chi=\sum_{|H|=|I|=p-1} M_{I, H}(z, \tau) \chi_{H}(\tau) d \tau_{I} .
$$

Comparison with the preceding relations shows that

$$
\begin{array}{r}
M_{I, H}(z, \tau)=\mathcal{Q}(z, \tau) \sum_{\left|H^{\prime}\right|=|J|=|K|=p-1} B_{I, K}\left(z_{0}, \tau\right) A_{H^{\prime}, J}(z, J(z) \tau) \\
\cdot \Gamma_{K, J}(z, J(z) \tau) \Gamma_{H, H^{\prime}}(z, J(z) \tau) .
\end{array}
$$

It is easy to give a global definition of the operator $M(z)$, and therefore of the form $\tilde{v}(z)$, in a full tubular neighborhood of $\mathfrak{L}_{z_{0}}$ by using a partition of unity subordinate to a covering $\left\{\omega_{i}\right\}_{i=1, \ldots, \nu}$ consisting of coordinate patches like $\omega$ above. We have $M\left(z_{0}\right)=$ Identity operator; and provided $\Delta$ is sufficiently small, $M(z)$ is invertible.

In summary we see that Equation (5.4) can be rewritten as

$$
\int_{\mathfrak{L}_{z_{0}}}(\tilde{v}(z), \varphi)_{\left(z_{0}\right)} d V\left(z_{0}\right)=0
$$

valid for all closed forms $\varphi \in \mathcal{C}_{c}^{\infty}\left(\mathfrak{L}_{z_{0}} ; \Lambda^{p-1}\right)$. Now that the analysis is concentrated on the fixed level set $\mathfrak{L}_{z_{0}}$ we can put $z=z(\sigma)$ and differentiate the equation (5.6) with respect to $\sigma>0$ :

$$
\int_{\mathfrak{L}_{z_{0}}}\left((\partial / \partial \sigma)^{l}[\tilde{v}(z(\sigma))], \varphi\right)_{\left(z_{0}\right)} d V\left(z_{0}\right)=0
$$

We have

$$
\begin{aligned}
(\partial / \partial \sigma)^{l} \tilde{v}(z(\sigma))= & M(z(\sigma))(\partial / \partial \sigma)^{l}\left[J(z(\sigma))^{*} v_{z(\sigma)}\right] \\
& +\sum_{l^{\prime}=1}^{l}\left(\begin{array}{c}
l \\
l^{\prime}
\end{array}\right)\left\{(\partial / \partial \sigma)^{l^{\prime}}[M(z(\sigma))]\right\}(\partial / \partial \sigma)^{l-l^{\prime}} J(z(\sigma))^{*} v_{z(\sigma)}
\end{aligned}
$$


Putting $\sigma=0$ and defining

$$
\begin{gathered}
\alpha^{(l)}=\left.(\partial / \partial \sigma)^{l} \tilde{v}(z(\sigma))\right|_{\sigma=0}, \\
\beta^{(l)}=\sum_{l^{\prime}=1}^{l}\left(\begin{array}{l}
l \\
l^{\prime}
\end{array}\right)\left\{\left.(\partial / \partial \sigma)^{l^{\prime}}[M(z(\sigma))]\right|_{\sigma=0}\right\}\left\{\left.(\partial / \partial \sigma)^{l-l^{\prime}}\left[J(z(\sigma))^{*} v_{z(\sigma)}\right]\right|_{\sigma=0}\right\},
\end{gathered}
$$

we conclude that Equation (5.3) does indeed hold.

Thanks to the estimates of the derivatives of $M(z)$ combined with induction on $l$ we may assume that good estimates of the tangential derivatives of $\beta^{(l)}$ and therefore of

$$
\begin{aligned}
& d_{\left(z_{0}\right)} \beta^{(l)}=\sum_{l^{\prime}=1}^{l}\left(\begin{array}{l}
l \\
l^{\prime}
\end{array}\right)\left\{\left.(\partial / \partial \sigma)^{l^{\prime}}[M(z(\sigma))]\right|_{\sigma=0}\right\}\left\{\left.(\partial / \partial \sigma)^{l-l^{\prime}}\left[J(z(\sigma))^{*} F_{z(\sigma)}\right]\right|_{\sigma=0}\right\} \\
& +\sum_{l^{\prime}=1}^{l}\left(\begin{array}{l}
l \\
l^{\prime}
\end{array}\right)\left\{\left.d_{\left(z_{0}\right)}(\partial / \partial \sigma)^{l^{\prime}}[M(z(\sigma))]\right|_{\sigma=0}\right\} \wedge\left\{\left.(\partial / \partial \sigma)^{l-l^{\prime}}\left[J(z(\sigma))^{*} v_{z(\sigma)}\right]\right|_{\sigma=0}\right\}
\end{aligned}
$$

have been established. Then Lemma 4.4 will give us good estimates of the tangential derivatives of $\alpha^{(l)}$. Furthermore (5.7) shows that $\alpha^{(l)}$ is orthogonal to all closed $(p-1)$-forms on $\mathfrak{L}_{z_{0}}$, i.e., $\alpha^{(l)}$ is the canonical solution of Equation (5.3).

We now return to the diffeomorphism $J(z)$. The hypothesis that the flow is tangent to the hypersurface $\Phi^{\pi}(x, t)=y$ is expressed by the equation

$$
\Phi_{x}^{\pi}(x, t) \dot{x}+\nabla_{t} \Phi^{\pi}(x, t) \cdot \dot{t}=\dot{y} ;
$$

while the hypothesis that the flow is transversal to the level sets $\mathfrak{L}_{z}$ is expressed by the equation

$$
\dot{t}=h \nabla_{t} \Phi^{\pi}(x, t) /\left|\nabla_{t} \Phi^{\pi}(x, t)\right|^{2},
$$

where $h$ is a real-valued $\mathcal{C}^{\infty}$ function of $\sigma \in\left[0, \sigma_{0}\right]$. Thus

$$
h+\Phi_{x}^{\pi}(x, t) \dot{x}=\dot{y} .
$$

As we have announced we shall let $z$ vary only along the horizontal line $x=x_{0}$ and the vertical line $y=y_{0}$; it suffices to choose $h$ in these two cases only.

Vertical motion $\dot{x}=0$. In this case we take $h \equiv 1$, which by (5.10) amounts to taking $\sigma=y: J\left(x_{0}+\iota y\right)$ is the gradient flow of $\Phi^{\pi}\left(x_{0}, t\right)$. We have

$$
\dot{t}=\nabla_{t} \Phi^{\pi}(x, t) /\left|\nabla_{t} \Phi^{\pi}(x, t)\right|^{2} .
$$

Horizontal motion $\dot{y}=0$. In this case we take $\sigma=x$, i.e., $\dot{x}=1$, whence, by (5.10), $h=-\Phi_{x}^{\pi}$ and

$$
\dot{t}=-\Phi_{x}^{\pi}(x, t) \nabla_{t} \Phi^{\pi}(x, t) /\left|\nabla_{t} \Phi^{\pi}(x, t)\right|^{2} .
$$

By $t(\sigma, \wp)$ we mean the solution of the systems of ODEs (5.11) and (5.12) such that $t(0, \wp)=\wp$. We can differentiate both sides of (5.11) and (5.12) with respect to $\sigma$ as well as in the directions tangential to $\mathfrak{L}_{z_{0}}$. To do this we return to the neighborhood $\omega$ of the "central" point $O \in \mathfrak{L}_{z_{0}}$ and we identify $\wp \in \mathfrak{L}_{z_{0}} \cap \omega$ to $\tau=\left(\tau^{\prime}, \tau_{n}\right)$ with $\tau^{\prime}=\left(\tau_{1}, \ldots, \tau_{n-1}\right), \tau_{n}=\psi\left(z_{0}, \tau^{\prime}\right)$; we write $t(\sigma, \wp)=t(\sigma, \tau)$; then $t(0, \tau)=\tau$. We let $(\partial / \partial \sigma)^{l} \partial_{\tau^{\prime}}^{\alpha}$ act on both sides of both differential equations (5.11) and (5.12); and we let $\partial_{\tau^{\prime}}^{\alpha}$ act on the initial value $\tau$. We have, for all $\tau \in \mathfrak{L}_{z_{0}} \cap \omega$,

$$
\left|(\partial / \partial \sigma)^{l} \partial_{\tau^{\prime}}^{\alpha} t(\sigma, \tau)\right| \leq \text { const. }\left|\nabla_{t} \Phi^{\pi}(x(\sigma), t(\sigma, \tau))\right|^{-k} .
$$


[To prove this estimate we use induction on $l+|\alpha|$ : for $l=0$ it follows by differentiating the equation $\Phi^{\pi}(x, t)=y$; for $l \geq 1$ by differentiating (5.11) or (5.12).] Freezing this estimate at $\sigma=0$ and applying the Lojasiewicz inequality (4.1) yields

Lemma 5.1. To each integer $k \geq 0$ there are numbers $B_{k}, \beta_{k}>0$, independent of $z_{0} \in \mathcal{U}$, such that, for all $l \in \mathbb{Z}_{+}, \alpha \in \mathbb{Z}_{+}^{n-1}, l+|\alpha| \leq k$, and all $\tau \in \mathfrak{L}_{z_{0}} \cap \omega$,

$$
\left.\left|\partial_{\sigma}^{l} \partial_{\tau^{\prime}}^{\alpha}[t(\sigma, \tau)]\right|\right|_{\sigma=0} \leq B_{k}\left[\operatorname{dist}\left(z_{0}, \mathcal{S}^{\pi}\right)\right]^{-\beta_{k}}
$$

We continue to view the $g_{i j}$ as functions of $(x, t)$. Lemma 5.1 implies

Lemma 5.2. To each integer $k \geq 0$ there are numbers $C_{k}, \gamma_{k}>0$, independent of $z_{0}$, such that, for all $i, j=1, \ldots, n-1, l \in \mathbb{Z}_{+}, \alpha \in \mathbb{Z}_{+}^{n-1}, l+|\alpha| \leq k$, and all $\tau \in \mathfrak{L}_{z_{0}} \cap \omega$,

$$
\left.\left\{\left|\partial_{\sigma}^{l} \partial_{\tau^{\prime}}^{\alpha}\left[g_{i j}(x(\sigma), t(\sigma, \tau))\right]\right|+\left|\partial_{\sigma}^{l} \partial_{\tau^{\prime}}^{\alpha}\left[g^{i j}(x(\sigma), t(\sigma, \tau))\right]\right|\right\}\right|_{\sigma=0} \leq C_{k}\left[\operatorname{dist}\left(z_{0}, \mathcal{S}^{\pi}\right)\right]^{-\gamma_{k}} .
$$

Proof. Letting $\partial_{\sigma}^{l} \partial_{\tau^{\prime}}^{\alpha}$ act on both sides of (4.5), putting $\sigma=0$ in the result and applying Lemma 4.1 yields an estimate

$$
\left|\partial_{\sigma}^{l} \partial_{\tau^{\prime}}^{\alpha}\left[g_{i j}(x(\sigma), t(\sigma, \tau))\right]\right|_{\sigma=0} \leq C_{k}\left[\operatorname{dist}\left(z_{0}, \mathcal{S}^{\pi}\right)\right]^{-\gamma_{k}},
$$

which entails a similar estimate for $g^{i j}$, by inversion and by (4.6), (4.7), (4.8).

The same approach allows us to estimate the partial derivatives (at $\sigma=0$ ) of the quantity

$$
\mathcal{Q}(z(\sigma), \tau)=\left[\operatorname{det}\left(g_{i j}(x(\sigma), t(\sigma, \tau))\right)\right]^{\frac{1}{2}}\left|\frac{D t(z, \tau)}{D \tau}\right|_{z=z(\sigma)}\left[\operatorname{det}\left(g_{i j}\left(x_{0}, \tau\right)\right)\right]^{-\frac{1}{2}} .
$$

To each integer $k \geq 0$ there are numbers $C_{k}, \gamma_{k}>0$, independent of $z_{0}$, such that, for all $l \in \mathbb{Z}_{+}, \alpha \in \mathbb{Z}_{+}^{n-1}, l+|\alpha| \leq k$, and all $\tau \in \mathfrak{L}_{z_{0}} \cap \omega$,

$$
\left.\left|\partial_{\sigma}^{l} \partial_{\tau^{\prime}}^{\alpha} \mathcal{Q}(z(\sigma), \tau)\right|\right|_{\sigma=0} \leq C_{k}\left[\operatorname{dist}\left(z_{0}, \mathcal{S}^{\pi}\right)\right]^{-\gamma_{k}} .
$$

We continue to estimate the various quantities in the expression of $M_{I, J}(z(\sigma), \tau)$ derived from (5.5). We limit our attention to $p \geq 2$, since $p=1 \Rightarrow M_{I, J}(z, \tau)=$ $\mathcal{Q}(z, \tau)$. If $J=\left\{j_{1}, \ldots, j_{p-1}\right\}, K=\left\{k_{1}, \ldots, k_{p-1}\right\}, 1 \leq j_{1}<\cdots<j_{p-1} \leq n-1$, $1 \leq k_{1}<\cdots<k_{p-1} \leq n-1$, then

$$
D_{J, K}^{\prime}(z(\sigma), t(\sigma, \tau))=\left(\partial \tau_{j_{1}} / \partial t_{k_{1}}\right) \cdots\left(\partial \tau_{j_{p-1}} / \partial t_{k_{p-1}}\right),
$$

where $\tau=\tau(\sigma, t)$ is obtained by solving the equation $t(\sigma, \tau)=t$ with respect to $\tau$. Since the Jacobian matrices

$$
\partial \tau^{\prime} / \partial t^{\prime}=\left(\partial \tau_{j} / \partial t_{k}\right)_{0<j, k<n}, \quad \partial t^{\prime} / \partial \tau^{\prime}=\left(\partial t_{j} / \partial \tau_{k}\right)_{0<j, k<n}
$$

are the inverse of one another, we have, for $1 \leq j, k \leq n-1$,

$$
\partial \tau_{j} / \partial t_{k}=\left(\operatorname{det} \partial t^{\prime} / \partial \tau^{\prime}\right)^{-1} D_{j k}
$$

where $D_{j k}$ is the appropriate minor of order $n-2$ in $\operatorname{det} \partial t^{\prime} / \partial \tau^{\prime}$. The upshot is that

$$
D_{J, K}^{\prime}(z(\sigma), t(\sigma, \tau))=\left(\operatorname{det} \partial t^{\prime} / \partial \tau^{\prime}\right)^{1-p} D_{j_{1} k_{1}} \cdots D_{j_{p-1} k_{p-1}} .
$$

If we take into account the fact that $\operatorname{det} \partial t^{\prime} /\left.\partial \tau^{\prime}\right|_{\sigma=0}=1$ we derive at once from Lemma 5.1 that to each integer $k \geq 0$ there are numbers $C_{k}, \gamma_{k}>0$, independent of $z_{0}$, such that, for all multi-indices $J$ and $K$ as above, all $l \in \mathbb{Z}_{+}, \alpha \in \mathbb{Z}_{+}^{n-1}, l+|\alpha| \leq$ $k$, and all $\tau \in \mathfrak{L}_{z_{0}} \cap \omega$,

$$
\left.\left|\partial_{\sigma}^{l} \partial_{\tau^{\prime}}^{\alpha}\left[D_{J, K}^{\prime}(z(\sigma), t(\sigma, \tau))\right]\right|\right|_{\sigma=0} \leq C_{k}\left[\operatorname{dist}\left(z_{0}, \mathcal{S}^{\pi}\right)\right]^{-\gamma_{k}} .
$$


Next we look at the entries $A_{I, K}$. Here also this only makes sense if $p \geq 2$. The $A_{I, K}$ are the coefficients of the Hermitian form $g_{(z)}^{*(p-1)}$ defined on covectors of degree $p-1$ by the metric $g_{(z)}$. In the coordinate patch $\left(\omega, t_{1}, \ldots, t_{n-1}\right)$ the Hermitian form $g_{(z)}^{*(1)}$ on cotangent vectors is defined by the equations

$$
g_{(z)}^{*(1)}\left(d t_{i}, d t_{j}\right)=g^{i j} \quad(1 \leq i, j \leq n-1) .
$$

It follows from this that, if $I=\left\{i_{1}, \ldots, i_{p-1}\right\}$ and $K=\left\{k_{1}, \ldots, k_{p-1}\right\}$ [with $1 \leq$ $\left.i_{1}<\cdots<i_{p-1} \leq n-1,1 \leq k_{1}<\cdots<k_{p-1} \leq n-1\right]$, then

$$
A_{I, K}=g^{i_{1} k_{1}} \cdots g^{i_{p-1} k_{p-1}} \text {. }
$$

It is then a direct consequence of Lemma 5.2 that to each integer $k \geq 0$ there are numbers $C_{k}, \gamma_{k}>0$, independent of $z_{0}$, such that, for all multi-indices $I$ and $K$ as above, all $l \in \mathbb{Z}_{+}, \alpha \in \mathbb{Z}_{+}^{n-1}, l+|\alpha| \leq k$, and all $\tau \in \mathfrak{L}_{z_{0}} \cap \omega$,

$$
\left.\left|\partial_{\sigma}^{l} \partial_{\tau^{\prime}}^{\alpha}\left[A_{I, K}(z(\sigma), t(\sigma, \tau))\right]\right|\right|_{\sigma=0} \leq C_{k}\left[\operatorname{dist}\left(z_{0}, \mathcal{S}^{\pi}\right)\right]^{-\gamma_{k}} .
$$

Similar estimates hold for the entries $B_{H, J}$ of the inverse matrix $\left(A_{I, K}\right)^{-1}$. In particular, if $|\alpha| \leq k$,

$$
\left.\left|\partial_{\tau^{\prime}}^{\alpha} B_{H, J}\left(z_{0}, \tau\right)\right|\right|_{\sigma=0} \leq C_{k}\left[\operatorname{dist}\left(z_{0}, \mathcal{S}^{1}\right)\right]^{-\gamma_{k}} .
$$

Combining the expression (5.5) with the estimates (5.13), (5.14), (5.15) and (5.16) yields similar estimates of the derivatives of $M_{I, J}$ with respect to $\left(\sigma, t^{\prime}\right)$, as needed. As indicated earlier this provides us with estimates of the derivatives of the right-hand side in (5.3), and thus, thanks to Lemma 4.4 applied with $f=F_{z_{0}}$, of the derivatives of the canonical solution $\alpha^{(l)}$, and thereby, of the tangential derivatives of $\left.(\partial / \partial \sigma)^{l}\left[J(z(\sigma))^{*} v_{z(\sigma)}\right]\right|_{\sigma=0}$. Taking into account the fact that $F$ is flat at the boundary of $\mathcal{O}$ enables us to complete the proof of Lemma 2.2.

\section{APPENDix: Estimates in THE INTEGRATION OF $p$-FORMS ON A COMPACT MANIFOLD}

We consider a compact, connected and orientable Riemannian manifold $(\mathcal{M}, g)$ of class $\mathcal{C}^{\infty}$, without boundary $(\operatorname{dim} \mathcal{M}=m \geq 1)$. All norms in the statements that follow (on tangent and cotangent spaces, on the Grassmann algebra, etc.) are defined by means of the metric on the base manifold. We denote by $\Lambda^{p}$ the $p$ th exterior power of the complexified cotangent bundle $\mathbb{C} T^{*} \mathcal{M}$. As usual $\Lambda^{0}$ is identified to $\mathcal{M} \times \mathbb{C}$, and $\Lambda^{p}=0$ if $p<0$ or if $p>m$; $d$ will stand for the exterior derivative in $\mathcal{M}$. We denote by $\mathcal{C}^{\infty}\left(\mathcal{M} ; \Lambda^{p}\right)$ the space of $\mathcal{C}^{\infty} p$-forms in $\mathcal{M}$, by $L^{2}\left(\mathcal{M} ; \Lambda^{p}\right)$ the Hilbert space of $L^{2} p$-forms in $\mathcal{M}$, and by $\|\cdot\|_{L^{2}}$ the norm in $L^{2}\left(\mathcal{M} ; \Lambda^{p}\right)$.

We restate Theorem 1.1 in [3]:

Lemma A.1. Suppose there exists an open covering $\left\{\mathcal{U}_{1}, \ldots, \mathcal{U}_{N}\right\}$ of $\mathcal{M}$ and two numbers $A>0$ and $r, 0<r \ll 1$, such that the following properties hold, for some $R>4^{m} r$ and every $i=1, \ldots, N$ :

$\exists t_{i} \in \mathcal{U}_{i}$ such that the exponential map $E_{i}: T_{t_{i}} \mathcal{M} \rightarrow \mathcal{M}$ is a diffeomorphism of the ball $B\left(t_{i}, R\right)=\left\{v \in T_{t_{i}} \mathcal{M} ;\|v\|<R\right\}$ onto $E_{i}\left(B\left(t_{i}, R\right)\right)$;

$$
A^{-1} \leq\left\|d E_{i}(v)\right\| \leq A \quad \text { for all } v \in B\left(t_{i}, R\right)
$$




$$
\mathcal{U}_{i}=E_{i}\left(B\left(t_{i}, r\right)\right) .
$$

Then there is a constant $C>0$ depending solely on $m$ and $A$, such that the following is true, for any integer $p, 1 \leq p \leq m$ :

If $f \in \mathcal{C}^{\infty}\left(\mathcal{M} ; \Lambda^{p}\right)$ is exact, then the equation $d u=f$ has a solution $u \in$ $\mathcal{C}^{\infty}\left(\mathcal{M} ; \Lambda^{p-1}\right)$ such that

$$
\|u\|_{L^{2}} \leq C r N^{4 p}\|f\|_{L^{2}} .
$$

Lemma A.1 yields an estimate for the canonical solution, that is, the solution orthogonal in $L^{2}\left(\mathcal{M} ; \Lambda^{p-1}\right)$ to all closed forms. Indeed this solution minimizes the $L^{2}$ norm over the affine subspace of all $L^{2}$ solutions.

\section{ACKNOWLEDGEMENTS}

We are indebted to Jean-Marie Trépreau for his patient reading and unrelenting criticism of the first three sections of the article, thereby bringing about many improvements. Needless to say all remaining shortcomings and errors remain the responsibility of the authors.

\section{REFERENCES}

1. M. S. Baouendi and F. Treves, A property of the functions and distributions annihilated by a locally integrable system of complex vector fields, Ann. of Math. 113 (1981), 387-421. MR 82f: 35057

2. S. Chanillo, The first eigenvalue of analytic level surfaces on spheres, Math. Research Letters 1 (1994), 159-166. MR 95g:58241

3. S. Chanillo and F. Treves, On the lowest eigenvalue of the Hodge Laplacian, J. Diff. Geom., to appear.

4. P. D. Cordaro and J. Hounie, On local solvability of underdetermined systems of vector fields, Amer. J. Math. 112 (1990), 243-270. MR 91j:58150

5. P. D. Cordaro and F. Treves, Homology and cohomology in hypo-analytic structures of the hypersurface type, J. Geometric Analysis I (1991), 39-70. MR 92h:32034

6. __ Hyperfunctions on Hypo-Analytic Manifolds, Annals of Math. Studies \# 136, Princeton University Press, Princeton, NJ, 1994. MR 96h:58163

7. L Necessary and sufficient conditions for the local solvability in hyperfunctions of a class of systems of complex vector fields, Invent. Math. 120 (1995), 339-360. MR 96j:58157

8. R. Hardt, Slicing and intersection theory for chains associated with real analytic varieties, Acta Math. 129 (1972), 75-136. MR 47:4110

9. 38 (1977), 207-217. MR 56:12302

10. L. Hörmander, Propagation of singularities and semi-global existence theorems for (pseudo)differential operators of principal type, Annals of Math. 108 (1978), 569-609. MR 81j:35110

11. _ Pseudo-differential operators of principal type, Nato Advanced Study Inst. on Singularities in Boundary Value Problems, Reidel Publ. Co., Dordrecht, 1981, 69-96. MR 83m:35003

12. S. Lojasiewicz, Sur les problème de la division, Studia Math. 18 (1959), 87-136. MR 21:5893

13. _ Ensembles semi-analytiques, IHES Notes, Paris, 1965.

14. G. Mendoza and F. Treves, Local solvability in a class of overdetermined systems of linear PDE, Duke Math. J. 63 (1991), 355-377. MR 92i:58177

15. L. Nirenberg and F. Treves, Solvability of a first-order linear partial differential equation, Comm. Pure Appl. Math. 16 (1963), 331-351. MR 29:348

16. Séminaire Schwartz, Unicité du problème de Cauchy. Division des distributions. Secrét. Math. Fac. Sci. Paris 4, 1959/60. MR 23:A2275

17. H. Sussmann, Real-analytic desingularization and subanalytic sets: an elementary approach, Trans. Amer. Math. Soc. 317 (1990), 417-461. MR 90e:32007

18. F. Treves, Study of a model in the theory of overdetermined pseudodifferential equations, Ann. of Math. 104 (1976), 269-324. MR 54:14014; MR 82i:35044 
19. - On the local solvability and local integrability of systems of vector fields, Acta Math. 151 (1983), 1-38. MR 85j:35009

20. _ Hypo-analytic Structures. Local Theory, Princeton University Press, Princeton, NJ, 1992. MR 94e:35014

21. R. Weitzenböck, Invariantentheorie, Groningen, Noordhoff, 1923.

Abstract. The article studies the local exactness at level $q(1 \leq q \leq n)$ in the differential complex defined by $n$ commuting, linearly independent realanalytic complex vector fields $L_{1}, \ldots, L_{n}$ in $n+1$ independent variables. Locally the system $\left\{L_{1}, \ldots, L_{n}\right\}$ admits a first integral $Z$, i.e., a $\mathcal{C}^{\omega}$ complex function $Z$ such that $L_{1} Z=\cdots=L_{n} Z=0$ and $d Z \neq 0$. The germs of the "level sets" of $Z$, the sets $Z=z_{0} \in \mathbb{C}$, are invariants of the structure. It is proved that the vanishing of the (reduced) singular homology, in dimension $q-1$, of these level sets is sufficient for local exactness at the level $q$. The condition was already known to be necessary.

Department of Mathematics, Rutgers University, New Brunswick, New Jersey 08903

E-mail address: chanillo@math.rutgers.edu

E-mail address: treves@math.rutgers.edu 